\title{
Prostatic Abscess: A Review and Update
}

\author{
Anthony Kodzo-Grey Venyo \\ North Manchester General Hospital, Department of Urology, Delaunays Road, Manchester, M8 5RB. United Kingdom.
}

Corresponding author: Anthony Kodzo-Grey Venyo, North Manchester General Hospital, Department of Urology, Delaunays Road, Manchester, M8 5RB. United Kingdom.

Received date: October 13, 2021; Accepted date: October 29, 2021; Published date: November 08,2021

Citation: Anthony Kodzo-Grey Venyo. (2021) Prostatic Abscess: A Review and Update. Archives of Medical Case Reports and Case Study, 4(6); DOI: $10.31579 / 2692-9392 / 090$

Copyright: (C) 2021 Anthony Kodzo-Grey Venyo, This is an open access article distributed under the Creative Commons Attribution License, which permits unrestricted use, distribution, and reproduction in any medium, provided the original work is properly cited.

\begin{abstract}
:
Prostate abscess (PA) is a complication that ensues an acute infectious purulent process within the prostate gland which is typified by accumulation of purulent material within the prostate gland and this purulent material could be unilocular or multi-septated within the prostate gland. PA is a rare disease especially in the developed world where PA tends to be more commonly associated with patients who have diabetes mellitus, chronic kidney disease, chronic liver disease, immunosuppressive disease, HIV Infection, renal transplantation, and long-term urethral catheterisation. PA tends to be more commonly encountered in the developing world. PA could be an acute abscess or chronic abscess especially in association with chronic inflammatory conditions including tuberculosis or intravesical instillations of Bacillus Calmette Guerin (BCG). PA tends to constitute $0.5 \%$ of all urology disease and $6 \%$ of all acute cases of bacterial prostatitis. The mortality rate associated with PA has tended to be between $1 \%$ and $16 \%$ of all cases of prostate abscess. PA tends to be most commonly encountered in individuals who are in their fifth to sixth decade and could occur at any age. PAs that are due to sexually transmitted organisms tend to be more commonly encountered in younger males. PA tends to affect the central zone as well as the peripheral zones of the prostate gland. Haematogenous dissemination from a primary source of infection from a primary infection elsewhere have been reported and some of the reported sources included respiratory tract, digestive tract, urinary tracts, skin, and soft tissue are very rare. PA has also been reported to be associated with staghorn calculus. Some of the reported bacterial organisms that have caused acute prostate abscess (APA) include: Escherichia Coli, Klebsiella, Pseudomonas, Proteus, Enterobacter, Enterococcus, and staphylococcus. Other causes of prostate abscess have included other bacteria and fungal infection including: Brucellosis, Salmonella, Nocardia, Mycobacterium tuberculosis. Prostate abscess has also been reported in association with malignancy of the prostate gland. Manifestations of prostate abscess could include: (a) systemic symptoms including pyrexia, chills, headache, general malaise, low back ache and in some rare cases of prostate abscess, a history of treatment for tuberculosis or contact with a person who has tuberculosis, or travel to a salmonella endemic area may be obtained and in cases of Brucellosis PAs drinking of raw milk / contact with animals may be elicited and or rare occasions treatment of superficial urothelial carcinoma with Bacillus Calmette Guerin may be elicited. (b) perineal pain, dysuria, urinary frequency, urinary urgency, retention of urine, recent prostate biopsy, visible haematuria, urethral discharge of pus, lower urinary tract obstructive symptoms with poor flow, intermittent flow, hesitancy, and sensation of incomplete emptying of the urinary bladder, retention of urine, and tenderness over the prostate gland with a feeling of bogginess and soft fluctuation. The symptoms tend to be non-specific. Diagnosis of PAs tends to be established based upon a good clinical history taking, good clinical examination, urinalysis and urine culture, routine haematology and biochemistry blood tests with evidence of raised white blood cell count and CRP and at times lymphocytosis in cases of tuberculous prostate abscess, blood culture, urine culture, PCR detection of sexually transmitted organisms, radiology imaging including trans-rectal ultra-sound scan of the prostate, or CT) scan of abdomen and pelvis including the prostate, or MRI scan of the prostate. Treatment of PA depending upon the size of the abscess has been undertaken with various options including: Appropriate antibiotic treatment alone for small abscesses based upon the antibiotic sensitivity pattern of the cultured organism, Antibiotic treatment and radiology image-guided aspiration of the abscess. Antibiotic treatment plus radiology image-guided insertion of a drain into the prostate abscess, trans-urethral incision / resection / modified resection of the prostate gland to deroof and drain the abscess, open drainage of the abscess is only undertaken on rare occasions.
\end{abstract}




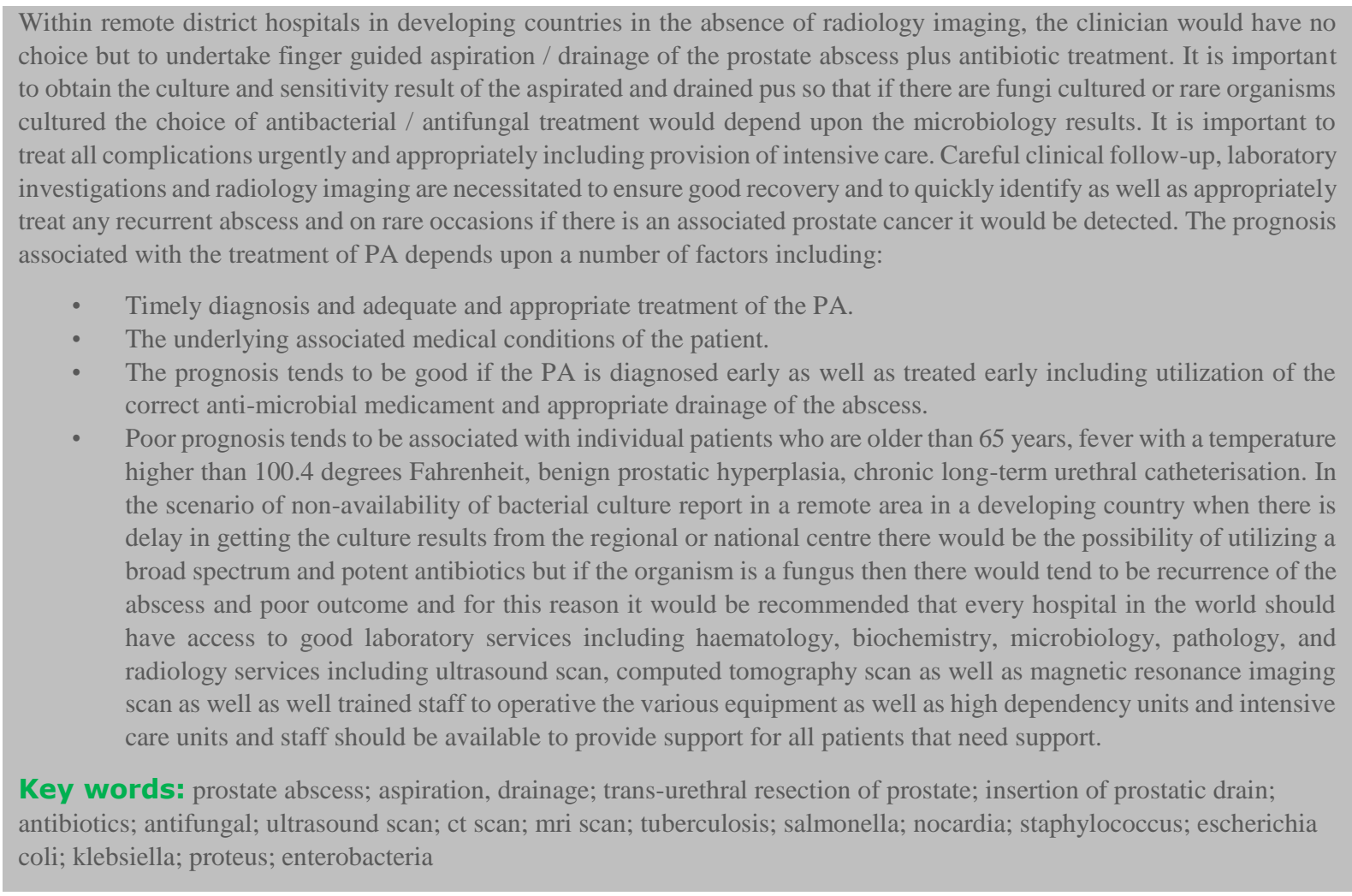

\section{Introduction:}

Reddivari and Mehta [1] made the ensuing summations related to an abscess and prostate abscess:

- An abscess usually tends represent an emanation of an inflammatory reaction to an infectious process and that an abscess is a terminology that is utilized for a collection of purulent material that tends to include cell debris, liquefied tissue from infective agents as well as enzymes.

- A prostate abscess is a terminology that is used for a localized collection of purulent fluid within the prostate gland, which quite often emanates as a complication of acute bacterial prostatitis (ABP).

- A prostate abscess tends to be difficult to differentiate acute bacterial prostatitis from a prostate abscess based upon the manifesting symptoms and clinical examination. [2]

- An abscess of the prostate gland could emanate in fatal consequences if appropriate measures are not taken, and this could possibly result in the development of severe urosepsis and septic shock. [3]

- With regard to the current day practice of medicine, the number of prostate abscess patients has reduced considerably in view of the judicious utilisation of antibiotics.

- Nevertheless, an abscess of the prostate gland tends to be relatively common in developing countries and prostate abscess tends to be relatively common in high-risk patients who have diabetes mellitus, end-stage renal disease (ESRD) patients who are on haemodialysis, liver cirrhosis, and immunodeficiency.

- It has been iterated that in view of the lack of clear global diagnostic and treatment guidelines, a prostate abscess could prove to be a challenge in the real world and prostate abscess could lead to significant morbidity. [4] Elshal et al. [5] made the following summations related to prostate abscess:

- It has been stated that abscess of the prostate gland tends to be an uncommon urological emergency but prostate abscess is a serious infection of the prostate gland which tends to be associated with a high mortality rate unless the prostate abscess is properly treated. [6] [7]

- $\quad$ Patients who have diabetes mellitus, renal insufficiency as well as immune suppression tend to have a particularly risk for the development of prostate abscess.

- It has also been iterated that urethral catheterisation, lower urinary tract instrumentation and a biopsy of the prostate gland are among the possible predisposing factors for the development of prostate abscess. [8]

- Many pathogens could be incriminated in the development of prostate abscess. Enterobacteriaceae (particularly Escherichia coli) and Staphylococcus aureus are the commonest causative organisms of prostate abscess [9]

- Haematogenous spread from distant foci had also been reported. In these cases, organisms like Mycobacterium tuberculosis and Candida species might be found [10]

- The clinical manifestation of prostate abscess does vary depending upon the severity of the infection.

- A prostate abscess usually tends to be diagnosed when a patient who has acute prostatitis does fail to respond to medical treatment.

- The patient who has prostate abscess commonly does manifest with perineal, genital and suprapubic pain, exacerbating lower urinary tract symptoms (LUTS), and urinary retention could also 
occur. Constitutional symptoms including fever, rigors, malaise and anorexia tend to be frequently present. Upon digital rectal examination, the prostate gland tends to be intractably tender. Fluctuation which is a 'boggy' sensation, of the prostate gland upon a digital rectal examination (DRE) could help establish the diagnosis of prostate abscess [11]

- It has been stated that trans-rectal ultrasound scan (TRUS) of the prostate gland as well as other cross-sectional radiology imaging methods including computed tomography (CT) scan of the pelvis or magnetic resonance imaging (MRI) scan of the pelvis could be useful with regard to the diagnosis, treatment and monitoring of the response to treatment of prostate abscess [12]

- Once liquefaction and abscess formation in the prostate have are diagnosed, many approaches to the drainage of the prostate abscess had described. Open perineal drainage, transurethral deroofing, transrectal needle aspiration or tube drainage. [13] [14] and percutaneous drainage procedures [15] have tended to be the main options of treatment for prostate abscess. Transurethral holmium-laser deroofing of a prostate abscess had also been reported in the literature [16]

- $\quad$ To the best of their knowledge, the available data do not support some treatments over other forms of treatment in any specific situation.

- Additionally, the morbidity of different treatment procedures for prostate abscess had not been sufficiently reported as well as the effect of different treatment options upon micturition as well as sexual function was not known.

- The need for the undertaking of secondary for the prostate abscess or for the treatment of the underlying pathology of the prostate gland had not been thoroughly assessed in the literature.

Considering that prostate abscess is a rare condition especially in the developed world as well radiology imaging facilities including ultrasound scan, magnetic resonance imaging (MRI) scans tend not to be readily available in remote district hospitals in some developing countries, there is the possibility that some clinicians may not be familiar with the diagnostic features and management options that are utilised to treat prostate abscesses. The ensuing article on prostate abscess is divided into two parts including: (A) Overview of general aspects of acute and chronic prostate abscess and (B) Miscellaneous narrations and discussions from some case reports, case series, and some studies related to prostate abscess including documentation of some of the organisms and conditions that have been related to the development of acute and chronic prostate abscess.

\section{METHODS}

Internet data search engines were searched including Google, Google Scholar, Yahoo, and PUBMED to obtain information on prostate abscess. The search words that were used included: Prostate abscess, prostatic abscess, abscess of the prostate, abscess of prostate gland, and prostate gland abscess, acute prostate abscess, chronic prostate abscess, candida prostate abscess, bacterial prostate abscess, fungal prostate abscess, Salmonella prostate abscess, and conditions associated with prostate abscess. Seventy two (72) references were obtained which were used to write the article in two parts including: (A) Overview of general aspects of acute and chronic prostate abscess and (B) Miscellaneous narrations and discussions from some case reports, case series, and some studies related to prostate abscess including documentation of some of the organisms and conditions that have been related to the development of acute and chronic prostate abscess.

\section{RESULTS}

\section{[(A)] Overview}

Some of the summations related to the overview of prostate abscess include: [17]

\section{Definition / general statements}

- It has been iterated that acute prostatic abscess is a complication that emanates from an acute infectious process of the prostate gland that is referred to as prostatitis which tends to be characterized by a focal accumulation of purulent material within the prostate gland [3]

- Majority of abscesses of the prostate gland tend to be acute abscesses; however, on very rare occasions chronic prostatic abscess has been documented for example tuberculous abscess of the prostate gland [18]. [19] [20] [21] [22]

- Other uncommon causes of prostatic abscess include Brucellosis in Brucellosis endemic areas especially in immunocompromised patients including diabetes mellitus [23] [24]

- Salmonella prostatic abscess has also been reported on rare occasions. [25] [26]

\section{Essential features}

- It has been iterated that acute bacterial prostatic abscess Infection tends to be caused mainly by gram negative rods [pathologyoutlines.com [17]

- It has been stated that in cases of acute prostatic abscess, neutrophils and necrotic debris tend to fill the prostatic ducts and acini [17]

Terminology

- It has been stated that with regard to terminology of acute bacterial prostatic abscess, the term acute inflammatory disease of the prostate gland has been utilized. [17]

\section{Epidemiology}

- It has been iterated that the incidence of acute prostatic abscess does represent $0.5 \%$ of all urological diseases and $6 \%$ of all acute bacterial prostatitis [17]

- It has been documented that the mortality rate associated with acute bacterial prostatitis has ranged between $1 \%$ and $16 \%$ [17]

- It has been iterated that acute prostatic abscess most commonly tends to affect men who are in their fifth to sixth decade; however, acute prostatic abscess can occur at any age [17]

- It has been iterated that older males tend to be affected by acute prostatic abscess in comparison with younger males. [17]

- It has been documented that prostatic abscess due to sexually transmitted organisms does occur more commonly in younger males in comparison with older males [4] [17] Sites

- It has been documented that acute prostatic abscess could affect the central and peripheral zones of the prostate gland [11] [17] 


\section{Pathophysiology}

- With regard to the pathophysiology of acute prostatic abscess, it has been iterated that The commonest way of developing the abscess is by means of reflux of infected urinary contents into prostatic ducts [17] [28].

- Risk factors for the development of prostatitis have been documented to include: an indwelling urinary catheter, underlying voiding dysfunction, poorly controlled diabetes mellitus, end stage renal disease, cirrhosis, and immunosuppression [17] [29]

- It has been stated that hematogenous dissemination of bacteria from a distant primary source of infection including septic foci in respiratory tract, digestive tract, and urinary tract, as well as the skin and soft tissue) could represent as rare source for the development of prostatitis. [17] [30]

\section{Aetiology}

- It has been stated that the most common bacterial causes of acute bacterial prostatic abscess include:: Escherichia coli which does account for greater than $70 \%$ of cases, Klebsiella, Pseudomonas, Proteus, Enterobacter, and Enterococcus species [3] [17] [31]

- It has been iterated that Staphylococcus aureus is a common cause of prostatic abscess which tends to be acquired through hematogenous dissemination [17] [30]

\section{Clinical Manifestations of acute prostatic abscess.}

The clinical presentations of acute prostatic abscess could divided into systemic symptoms and localised prostatic symptoms as follows: [17]

- Systemic Manifestations [6]

○ Headache, fever, chills and general malaise/fatigue, and low back pain

- Prostate Localised Manifestations [14]

Some of the localised symptoms of acute prostatic abscess include:

- Severe tenderness of the prostate gland upon digital rectal examination with areas of fluctuation

○ Pain within the perineum

○ Dysuria, urinary urgency or urinary frequency, haematuria, purulent urethral discharge

- Obstructive lower urinary tract symptoms including hesitancy, poor urinary flow, intermittent urinary flow, and incomplete emptying of the urinary bladder.

\section{Diagnosis}

- History and physical findings

- Complete blood count with differential, urinalysis

- Imaging with transrectal ultrasound (the diagnostic method of choice), CT scan, and MRI scan tend to be rarely used. [14]

- Blood culture, urine culture

- PCR (detection of sexually transmitted organisms)

\section{Laboratory}

- > 10 white blood cells per high power field and pyuria
- Bacterial culture and antibiotic susceptibility identification

- DNA sequencing and nucleic acid amplification by PCR

- Can raise the serum prostate specific antigen (PSA) above normal [32]

\section{Radiology description}

- Transrectal ultrasound (TRUS) [14]

○ 1 or more hypoechoic areas with well-defined walls

- Thick pus primarily in the transition zone and in the central zone of the prostate

- Computed tomography (CT scan) of the abdomen and pelvis. [33]

○ Can better delineate the spread of infection to adjacent organs

- Magnetic resonance imaging (MRI) [34]

- Hypointense signal on T1 and hyperintense on T2 image

\section{Differential Diagnoses}

Some of the differential diagnoses of prostate abscess include:

- Acute prostatitis

- Chronic prostatitis

- Granulomatous prostatitis

- Carcinoma of the prostate gland

[(B)] Miscellaneous narrations and discussions from case reports, case series, and studies related to acute prostatic abscess, and chronic prostatic abscess.

Elshal et al. [35] assessed the outcome of the drainage procedure that was used for the treatment of a prostatic abscess, in order to propose a treatment algorithm to reduce the morbidity and the need for re-treatment of prostate abscess. With regard to the patients and methods, Elshal et al. [35] retrospectively reviewed patients who had been admitted and who had undergone an interventional treatment for a prostate abscess. Elshal et al. [35] reviewed all baseline relevant variables. Elshal et al. [35] recorded details of the intervention, laboratory data, duration of hospital stay, follow-up data as well as re-admissions of the patients. Elshal et al. [35] summarised the results as follows:

- A prostate abscess was diagnosed in 42 patients and out of these 42 patients, 30 patients were treated by means of trans-urethral deroofing and 12 patients were treated by means of trans-rectal needle aspiration of the prostate abscess. The size of the abscess had measured between $2 \mathrm{~mL}$ and $23 \mathrm{~mL}$ with a median size of $4.5 \mathrm{~mL}$, and a size that ranged between $1.5 \mathrm{~mL}$ and $7.1 \mathrm{~mL}$ with a median size of $2.7 \mathrm{~mL}$ in the deroofing and aspiration groups, respectively $(P=0.2)$.

- With regard to half of the cases multiple abscesses were evident upon radiology imaging before the intervention procedure.

- The median (range) of hospital stay following deroofing procedure and aspiration of the prostate abscess was $2(1-11)$ days and $1(1-19)$ days, respectively $(P=0.04)$.

- $\quad$ Perioperative complications had occurred only in the deroofing group, in which two patients did develop septic shock which required intensive care management (Clavien 4) and one patient developed epididymo-orchitis (Clavien 2). There were two late complications within the deroofing group, in which one patient did develop a urethral stricture which required endoscopic urethrotomy (Clavien 3a) and one patient developed a urethral diverticulum and urinary incontinence which required 
treatment by diverticulectomy and a bulbo-urethral sling procedure (Clavien 3b). A urethro-rectal fistula did develop following after aspiration of the prostate abscess in one patient. Re-treatment for the abscess was necessitated in two (7\%) patients in the deroofing group, which was treated by means of aspiration of the abscess.

Elshal et al. [35] concluded that:

- Transrectal needle aspiration of a prostate abscess, when undertaken for properly selected cases, could minimise the morbidity of the drainage procedure.

Lee et al. [3] undertook a study in order to ascertain the risk factors that tend to be associated with the formation of abscess in acute bacterial prostatitis, as well as to compare the outcomes of treatment between abscess group and non-abscess group. Lee et al. [3] undertook a multicentre retrospective cohort study in which all patients who were suspected to have an acute infection of the prostate gland underwent computed tomography $(\mathrm{CT})$ scan or trans-rectal ultrasound scan of the prostate gland to differentiate acute abscess of the prostate gland from acute prostatitis without abscess formation. Lee et al. [3] summarised the results as follows:

- A total of 31 prostate abscesses were reviewed among 142 patients who had acute prostatitis.

- Univariate analysis of the cases demonstrated that the duration of symptom, diabetes mellitus as well as voiding disturbance represented predisposing factors for abscess formation in cases of acute prostatitis.

- Nevertheless, diabetes mellitus was found not to be related to prostate abscess in multivariate analysis.

- $\quad$ Patients whose abscesses measured less than $20 \mathrm{~mm}$ in size had not undergone surgery and they were cured without any complications.

- On the contrary, patients whose abscesses measured more than $20 \mathrm{~mm}$ who had undergone transurethral resection of the prostate gland (TURP) had a shorter duration of antibiotic treatment in comparison with those who did not have surgery.

- Irrespective of surgical treatment, both the length of hospital stay and antibiotic therapy were longer with regard to patients who had prostatic abscesses than they were in those without abscesses.

- Nevertheless, the incidence of septic shock was not different between the two groups.

- A wide spectrum of microorganisms was responsible for prostate abscesses. In contrast, Escherichia coli was found to be the predominant organism responsible for acute prostatitis without abscess.

Lee et al. [3] made the ensuing conclusions:

- $\quad$ Radiology imaging studies should be considered when patients who have acute prostatitis have delayed treatment and signs of voiding disturbance.

- Early diagnosis of prostatic abscess tends to be beneficial because prostatic abscesses do require prolonged treatment protocols, or even they do require surgical drainage.

- Surgical drainage procedures such as transurethral resection of the prostate gland were not necessary in all patients with prostate abscesses.
- Nevertheless, surgical intervention could portend potential merits that reduce the antibiotic exposure period and does tend to enhance voiding function in patients who have prostatic abscess.

\section{Abdelmoteleb et al. [4] iterated the following:}

- With regard to contemporary practice, the number of patients who manifest with prostatic abscess have significantly reduced due to the widespread utilisation of antibiotics.

- Nevertheless, when faced with the pathology, prostatic abscess tends to pose a challenge to clinicians in view of the difficulty of establishing the diagnosis and lack of guidelines for treatment of the abscess.

- The treatment of prostatic abscess does tend to consist of an array of measures including utilization of parenteral broadspectrum antibiotic administration and drainage of the abscess.

Barozzi et al. [11] stated that prostatic abscesses tend to be uncommon within clinical practice in view of the fact that utilisation of early antibiotic treatment has led to the reduction of complications of prostatitis. Barozzi et al. [11] also stated that prostatic abscess does mainly affect diabetic and immunosuppressed patients and that the organisms which most frequently tend to be involved in acute prostatic abscess tend to include: Escherichia coli and Staphylococcus, whereas gonococcus rarely tends to be encountered. Barozzii et al. [11] reported that the results in eight men with prostatic abscess they had managed showed that five of the men were treated with ultrasound-scan-guided percutaneous drainage of the abscess. They also reported that the diagnosis of prostatic abscess was clinically suspected in only three patients, and the diagnosis of prostatic abscess was confirmed by transrectal ultrasound scan of the prostate gland. All patients experienced complete resolution of their prostatic abscess. Barozzi et al. [11] made the following conclusions:

- Trans-rectal ultrasound scan of the prostate gland is the most reliable radiology imaging method to diagnose prostatic abscess.

- Percutaneous trans-perineal or trans-rectal drainage of the prostatic abscess is the first choice for the treatment of prostatic abscess in view of the lower risk of complication in comparison with surgery.

Tiwari et al. [28] undertook a retrospective study which was aimed to ascertain the clinical findings as well as treatment strategies in 24 patients who had been admitted due to prostatic abscess from 1999 to 2008. Tiwari et al. [28] reported that the diagnosis of prostate abscess was established clinically based upon digital rectal examination that demonstrated presence of positive fluctuation and tenderness within the prostate gland. Tiwari et al. [28] additionally stated that all cases were confirmed based upon trans-rectal ultrasound (TRUS) of the prostate gland and only positive cases were included in the study. Tiwari et al. [28] undertook the following diagnostic work-up assessment of the patients: analysis of midstream specimens of urine of the patients as well as analysis abscess fluid culture for pathogens. The treatment options that were used included: (a) endoscopic trans-urethral incision of the prostate (TUIP), or (b) transperineal aspiration of the abscess under ultrasound scan-guidance or (c) conservative treatment. With regard to the results, Tiwari et al. [28] stated the following:

- Out of the 24 patients that were studied, $45.83 \%$ of the cases did have a pre-disposing factor, and diabetes mellitus which constituted $37.50 \%$ of the cases was the commonest. 
- Digital rectal examination demonstrated fluctuation in $70.83 \%$ of the cases.

- Trans-abdominal ultrasound scan did miss the prostatic abscess in $29.18 \%$ of the cases.

- Upon trans-rectal ultrasound (TRUS) scan of the prostate gland, the ultrasound scan of all the study patients did demonstrate hypo-echoic zones, while nine others did demonstrate internal septations.

- With regard to majority of the cases, the lesion was peripheral.

- A causative pathogen could be identified in $70.83 \%$ of the prostatic abscesses.

- Surgical drainage of the abscess by means of trans-urethral deroofing was undertaken in 17 cases that included one case of failed aspiration. Trans-perineal aspiration of the prostatic abscess under trans rectal ultrasound (TRUS) scan-guidance was undertaken in three cases and conservative treatment with follow-up was adopted in five cases.

Tiwari et al. [28] made the ensuing iterations:

- Their data did confirm the importance of predisposing factors in the pathogenesis of prostatic abscess.

- In majority of the cases, the clue to the diagnosis of prostatic abscess was obtained by digital rectal palpation of the prostate gland.

- Trans-recta ultrasound (TRUS) scan does give the definite diagnosis of prostatic abscess and also does help in follow-up of patients who have been treated for prostatic abscess.

- Trans-urethral deroofing of the prostate gland is the ideal treatment when the abscess cavity is more than $1 \mathrm{~cm}$, although in some selected cases, trans-rectal ultrasound TRUS)-guided aspiration of the prostatic abscess or conservative treatment does have a role to play in the treatment of prostatic abscess.

Brede et al. [29] made the ensuing iterations about acute prostatitis, prostatic abscess, and prostatic fistula:

- Acute bacterial prostatitis is a common as well as a clinically important genitourinary disorder.

- The population of patients who are at especially high risk for the development of acute prostatitis do include those who have diabetes mellitus, cirrhosis, as well as suppressed immune systems.

- The cause of acute bacterial prostatitis tends usually to be an ascending infection, however, bacteria could also be introduced during trans-rectal biopsy of the prostate gland.

- Clinical manifestation of acute bacterial prostatitis does range from mild lower urinary tract symptoms to full sepsis.

- The causative organisms acute bacterial prostatitis tend to be usually similar to those that cause other common genitourinary infections, and these include Escherichia coli and Enterococcus spp.

- Oral or intravenous antibiotics usually tend to effective for curing the acute prostatitis infection and the progression the acute infection to chronic bacterial prostatitis, therefore tends to be uncommon.
- Patients who are immunosuppressed do require special consideration, as bacterial prostatitis in such patients could be caused by atypical infecting organisms and these infections might, therefore, require additional treatments.

- A lack of response to standard treatment for acute prostatitis could lead to complications such as a prostatic abscess or fistula.

Susaníbar Napurí et al. [30] reported two cases of prostate abscess that had difficult management. They reported two patients who had prostatic abscess as follows: The first patient was a 73-year-old diabetic man, who was treated with utilization of a more passive approach with percutaneous trans-rectal drainage of the abscess fluid and after a slow response, the patient died as a result of developing sepsis. The second patient was a 59-year-old man who did experience a negative clinical response to antibiotic treatment of his prostate abscess. While he was undergoing antibiotic ambulatory care, the patient underwent transurethral resection of his prostate gland which did yield a successful outcome. Susaníbar Napurí et al. [30] made the following conclusions:

- Prostate abscess is a clinical rare entity which does affect individuals that experience weakness and it can be a serious condition.

- Measures taken to arrive at a resolution of the prostate abscess must be rapid and appropriate.

Schneider et al. [31] stated the following:

- $\quad$ During the last years tremendous changes had occurred with regard to the epidemiology knowledge and the diagnostic process of the prostatitis syndrome.

- A new globally-accepted classification system of prostatitis had become the gold standard in contemporary literature.

Schneider et al. [31] undertook a study to compare the inflammatory and infectious status of men who have prostatitis syndrome with results from their study cohort from 1992. A total of 168 symptomatic men, whose ages had ranged between 18 years and 79 years whose mean age was 43.2 years that attended the Giessen prostatitis outpatient department were included in the study. All men had undergone a standard four-glass-test including leucocyte analysis in all specimens. A routine search for Ureaplasma urealyticum and Chlamydia trachomatis was undertaken. Ejaculate analysis following World Health Organization (WHO) criteria was undertaken including the evaluation of increased number of peroxidase-positive leucocytes (PPL). The men were classified according to the National Institutes of Health $(\mathrm{NIH})$ prostatitis classification. The distribution of patients according to NIH criteria was as follows: NIH II (4.2\%), NIH IIIA (31.5\%), NIH IIIB (50.0\%) and urethra-prostatitis $(14.3 \%)$. Chlamydial infection was present in one man (0.6\%). Only two men with increased leucocytes within prostate secretions demonstrated greater than or equal to 106 million ml-1 PPL in semen. As compared with the cohort study 10 years earlier,, the proportion of the different subtypes of the prostatitis syndrome had remained stable. The aetiological spectrum of chronic bacterial prostatitis had not changed, however, in contrast, the prevalence of $\mathrm{C}$. trachomatis was then found to be strikingly reduced. Using the WHO cut-points for leucocytospermia the inclusion of seminal leucocytes to the diagnostic process had not influenced the distribution between inflammatory (type NIH IIIA) and noninflammatory (type NIH IIIB) chronic pelvic pain syndrome. The lesson learnt from this summation is that some rare organisms could also be involved in prostatitis syndrome and prostatic abscess that emanate from the prostatitis syndrome and clinicians need to be aware that on rare occasions they may need to treat chlamydia abscesses of the prostate gland with an 
appropriate antibiotics pursuant to drainage or surgical treatment of prostatic abscesses.

Jang et al. [14] stated that prostate abscess is an uncommon urological disease but it does tend to have a high mortality rate if it is not treated properly. They also stated that the diagnosis and proper treatment of prostatic abscesses had remained a challenge for physicians. Jang et al. [14] compared data on conservative treatments, transurethral resection of prostatic abscess, and transrectal ultrasound (TRUS)-guided needle aspiration in 52 cases over a period of 10 years. With regard to materials and methods, Lang et al. [14] reported that the records of 52 patients who were diagnosed as having prostate abscess based upon computed tomography at Gangnam Severance Hospital between January 2000 and September 2010 were retrospectively reviewed. All of the patients were discharged when their leucocytosis had normalized and the patients had been free of fever for 2 days. Multivariate regression analysis was undertaken to ascertain independent risk factors for the length of hospitalization. Jang et al. [14] summarised the results as follows:

At the time of the diagnosis of prostatic abscess, the ages of the 52 patients had ranged between 33 years and 81 years and the mean age was 56.3 years. The average volume of the prostate was $56.3 \mathrm{ml}$ and the volume of the prostate gland had ranged between 21 and $223 \mathrm{ml}$ ), the average prostate-specific antigen level was $18.54 \mathrm{ng} / \mathrm{ml}$ which ranged between 2.0 $\mathrm{ng} / \mathrm{ml}$ and $57.0 \mathrm{ng} / \mathrm{ml}$, and the average size of the abscess was $3.8 \mathrm{~cm}$ as well as the size of the prostate abscess had ranged between 2.1 and 5.5 $\mathrm{cm}$.. All patients were treated by means of parenteral antibiotics during their hospital stay with intravenous antibiotics that entailed fluoroquinolone monotherapy or 3rd-generation cephalosporin in combination with an aminoglycoside. Out of the 52 patients, 22 had diabetes mellitus that amounted to $42.3 \%, 19$ of the patients had hypertension that amounted to $36.5 \%$ of the patients, and 7 patients that amounted to $13.5 \%$, had paraplegia due to spinal cord injury. The most common manifestations were fever in $47,90.4 \%$ of the patients, perineal discomfort in $43,82.7 \%$ of the patients, dysuria in $40,76.9 \%$ of the patients, and urinary retention in $29,55.8 \%$ of the patients. The prostate abscesses were treated by means of conservative treatment in 11 cases, transurethral resection of prostatic abscess in 23 cases, and TRUS-guided needle aspiration of the abscess in 18 cases.. The average hospitalization stay was 17.5 days and this did range from 6 days to 39 days. The average hospitalization stay of conservative treatment patients was 19.1 days and did range from 9 days to 39 days. Patients who underwent treatment by transurethral resection of prostatic abscess and TRUS-guided needle aspiration stayed for an average of 10.2 days but this did range between 6 days and 15 days; and an average stay of 23.25 days which did range, from 18 to 34 days, respectively. Of the 18 cases who had undergone needle aspiration, the prostate abscesses recurred in 4 cases that amounted to $22.2 \%$ within 1 month pursuant to the discharge of the patients. The 2 patients who had conservative treatment died due to sepsis. Jang et al. [14] did not find any independent factors that affected the average hospitalization period. Jang et al. [14] concluded that:

- Patients who have prostate abscess who were treated by means of transurethral resection of the prostate had a significantly shorter hospitalization length in comparison with needle aspiration patients.

Thornhill et al. [33] studied the value of computed tomography (CT) scan of the prostate and pelvis as well as ultrasound scan of the prostate gland with regard to the diagnosis and follow-up of prostate abscesses that they had studied in 6 patients who had prostate abscess. With regard to the results, Thornhill et al. [33] reported that five patients had CT scan alone, one patient had had CT scan and ultrasound scan and one patient had ultrasound scan only. Thornhill et al. [33] also reported that the CT scan findings did include an enlarged prostate gland with non-enhancing fluid density collections which sometimes were multiseptated or which had enhancing rims. The ultrasound scan findings were reported to be similar in that they demonstrated a hypo-echoic mass that had thick walls. Follow-up examinations pursuant to antibiotic treatment in which one CT scan and one ultrasound scan were undertaken showed improvement or resolution. Thornhill et al. [33] concluded that:

- In the patients who were studied, CT scan and ultrasound scan were useful methods to detect and follow the course of prostatic abscess.

Singh et al. [34] stated that DIFFUSION: weighted MRI (DWI) is a novel technique which is utilised to analyse the diffusion of water molecules in vivo. And that DWI has been utilised extensively in the central nervous system. As well as they stated that Its use in body imaging is on the rise and that with regard to the prostate gland, it has been utilised in the evaluation of prostatic carcinoma. Singh et al. [34] reported the DWI findings in two patients of prostate abscess and they pointed out the usefulness of MRI scan in the assessment and diagnosis of prostate abscess.

Jana et al. [36] stated that abscesses of the prostate gland usually tend to be related to gram negative bacilli. Nevertheless, methicillin-resistant Staphylococcus aureus (MRSA) had emerged as a substantial cause of prostate abscesses over recent years. Jana et al. [35] reported the case of a 31-year-old man who had a history of orthotopic liver transplantation 10 years preceding the report of the case, who had manifested with acute onset of dysuria and abdominal pain and who was diagnosed as having an MRSA prostate abscess. They stated that to their knowledge, the reported case was the first case that had described a prostate abscess in a liver transplant recipient and the first case that reported MRSA as the causative organism of a prostate abscess in a solid organ transplant recipient. A lesson learnt from this short summation is the fact that even though rare, methicillin resistant staphylococcus aureus could cause prostate abscess and therefore clinicians who treat patients who have prostate abscess should ensure that they see the culture and sensitivity results of all cases of prostate abscess fluid they send for microbiology examination to ensure they are aware of the causative organism of the abscess and the sensitivity pattern of the cultured organism to enable them treat their patients with the most appropriate antibiotics to ensure complete resolution of the abscess and to avoid or reduce the incidence of recurrent prostate abscess.

Carroll et al. [36] stated that prostate abscess is an uncommon complication of acute bacterial prostatitis and it is most commonly caused by Enterobacteriaceae. Carroll et al. [36] reported on a case of prostate abscess which was caused by Staphylococcus aureus that was successfully treated by means of a combination of antibiotic and surgical treatment. The isolate was non-multidrug-resistant, methicillin-resistant Staphylococcus aureus and which was genotyped as clonal complex 5, an emerging regional clone that tends to be trimethoprim resistant and Panton-Valentine leukocidin positive. Carroll iterated that their current reported case was the first case to report the use of clindamycin step-down treatment. Carroll et al. [36] iterated that their review of the literature had identified a further 39 cases of Staphylococcus. aureus prostate abscesses, of which 26 were methicillin resistant. Carroll et al. [36] made the following conclusions:

- Staphylococcus. aureus is a rare cause of prostate abscess.

- Optimal management of Staphylococcus prostate abscess does include both antibiotic treatment and surgical drainage of the abscess.

- Their use of clindamycin as step-down treatment was guided by its excellent prostatic penetration. 
Liao et al. [37] stated the following: (a) Prostate abscess usually tends to be a complication of acute urinary tract infection. (b) Invasive liver abscess syndrome is characterized with Klebsiella pneumoniae related multiple organ metastasis. (c) Concomitant pyogenic liver abscess and prostate abscess had rarely been reported in the literature. (d) Recurrent episode of liver abscess is even rarer. Liao et al. [37] reported a 71-yearold man who developed acute bacterial prostate abscess and urinary tract infection that was caused by Klebsiella. pneumoniae which was associated with multiple liver abscess, psoas muscle abscess and osteomyelitis. The patient's blood culture and urine culture yielded Klebsiella. pneumoniae, that confirmed the diagnosis of invasive liver abscess syndrome which was caused by Klebsiella. pneumoniae. The patient was successfully treated with empirical antibiotics for 6 weeks. Liao et al. [37] concluded that their reported case had emphasized the importance of timely and accurate diagnosis which should be ensued by appropriate treatment in disseminated Klebsiella. pneumoniae infection in order to prevent significant morbidity and mortality.

Bastide et al. [38] stated that fungal urinary tract infection does represent a high-risk event in severely ill patients. Bastide et al. [38] reported a case of a prostate abscess due to Candida tropicalis which was not associated with any systemic manifestations. They reported that on the first occasion, a conservative treatment with antifungal treatment and transrectal ultrasound-guided drainage was performed without success. In view of this transurethral resection of the prostate was required for drainage with a favourable course.

Somuncu et al. [39] stated prostatic abscess is uncommon but; however, serious disease and that prostatic abscess should be diagnosed at an early stage by the combination of clinical examination and transrectal ultrasound, as well as drained. Somuncu et al. [39] treated a 79-year-old man who had multiple prostate abscesses (PAs) by utilising lavage of the saline and antibiotic (cefoxitin) pursuant to transrectal ultrasound-guided transrectal puncture and aspiration of the abscess. Somuncu et al. [39] reported that that at his 12 months follow-up there was no evidence of recurrence of his prostate abscess based upon his clinical examinations and ultrasound scan follow-up assessments over the period of one year. .

Chou et al [40] undertook a study to analyse the trans-rectal ultrasound (US), or TRUS and colour Doppler ultrasonography (CDU) findings as well as treatment strategies with TRUS-guided procedures in 13 patients who had prostate abscess. They reported that over a period of 6 years, 18 prostate abscesses had been diagnosed in 13 patients whose mean age was 59 years. They reported that the diagnostic work up of the patients did include TRUS, analysis of mid-stream specimens of urine, as well as analysis, as well as analysis and culture of the prostate abscess fluid for leucocytes and pathogens. With regard to the treatment of the patients, the patients were treated either by a conservative treatment option with regard to abscesses that were less than $1 \mathrm{~cm}$ in diameter, or by means of aspiration of the prostate abscess or drainage of the prostate abscess of the abscesses were either $1 \mathrm{~cm}$ or greater than $1 \mathrm{~cm}$ in diameter. Chou et al. [40] corelated the CDU findings to the treatment effects. Chou et al. [40] also reviewed the predisposing factors for the development of the prostate abscess. They reported that with regard to the 13 patients, the commonest clinical manifestations were urinary frequency in $77 \%$ of the patients, and pus cells within the mid-stream urine in $92 \%$ of the patients. Predisposing factors were found in 11 men, and 5 of the men had diabetes mellitus. With regard to 10 patients, the definitive peri-interventional diagnosed was based upon trans rectal ultrasound scan (TRUS) findings. TRUS with probe palpation did demonstrate tiny floating echogenic speckles within the abscess cavity with regard to 4 patients. CDU had demonstrated increased colour-flow signals at the margin and encompassing tissue of the abscess pockets. Abscesses that had poorly defined boundaries did have more prominent surrounding colour-flow signals and had achieved, with relative difficulty, a satisfactory aspiration procedure. Aspiration was undertaken for all 11 abscesses that measured between $1 \mathrm{~cm}$ and 3 $\mathrm{cm}$. A total of 4 larger abscesses that measured more than $3.0 \mathrm{~cm}$ were treated with aspiration or drainage utilizing a 5-French pigtail catheter. No surgical drainage was undertaken. Chou et al. [40] made the ensuing iterations:

Trans-rectal CDU could help in the evaluation of maturity of an abscess pocket. US-guided aspiration with an 18-gauge needle or drainage with a 5-French pigtail catheter significantly shortened the hospital stay.

Varkarakis et al [41] undertook a study to evaluate the feasibility and effectiveness of three-dimensional ultrasound-guided trans-perineal puncture and drainage of prostate abscesses. They reported that between 1985 and 2003, a prostate abscess was diagnosed in 22 men. One of the treatment options used to drain the abscesses was trans-perineal puncture under three-dimensional ultrasound scan-guidance. They compared the post-operative results with the outcomes of other modalities. With regard to the results, Varkarakis et al. [41] reported that the average age of the patients was 52 years and predisposing factors were found in 12 patients. Trans-perineal puncture of the prostate abscess was undertaken in 7 patients under general anaesthesia. In addition to antibiotic treatment, a nephrostomy tube was left in place in all of the patients for a mean period of 3 days to drain the prostate abscess. All of the patients were treated effectively without any additional treatment or complications. Varkarakis et al. [41] concluded that three-dimensional ultrasound scan-guided transperineal puncture was a minimally invasive and effective technique to be utilized for the treatment of prostate abscesses.

Basiri eand Jayaherforooshzadeh [42] stated that prostate abscess is an uncommon infection which can be treated by antibiotic administration and drainage and the reported mortality rate of the prostate abscess is between $3 \%$ and $16 \%$. They also stated that with regard to patients who are refractory to medical treatment, surgical interventions are advocated. They reported a patient with the prostate abscess who was treated with percutaneous drainage. Basiri et al. [42] reported a 67-year-old man who had obstructive lower urinary symptoms and prostate-specific antigen level of $8.9 \mathrm{ng} / \mathrm{mL}$ who had undergone trans-perineal prostate biopsy. Three days subsequently, the patient manifested as an the emergency with fever, chills, and poor general condition. His clinical examination demonstrated that his blood pressure, pulse rate, and temperature were $130 / 80 \mathrm{mmHg}, 105$ per minute, and $39^{\circ} \mathrm{C}$, respectively. He had a digital rectal examination which demonstrated that he had a huge enlarged prostate with a prominent left lobe. He had urine and blood cultures, preceding the commencement of his antibiotic treatment and both cultures exhibited Escherichia coli. He had trans-abdominal ultrasound scan of the prostate gland which demonstrated a hypo-echoic mass that contained abundant debris within his left lobe of the prostate gland. He had computed tomography (CT) scan which showed a $5 \mathrm{~cm} \times 8 \mathrm{~cm}$ homogenous mass that had a low density upon the same region of his prostate gland. He received vancomycin, metronidazole, and ceftazidime intravenously. He subsequently underwent percutaneous drainage of the prostate abscess in view of his unresponsiveness to his medical treatment. His prostate abscess was drained transperineally with utilization of transrectal ultrasonography (TRUS) guidance and a suprapubic cystostomy was undertaken. A Pigtail catheter was inserted into the abscess cavity and irrigation of the abscess cavity was undertaken twice per day with utilization of saline and antibiotics. On the 5th day, following discontinuation / stoppage of discharge of pus from his drainage catheter, the Pigtail catheter and the cystostomy were removed. One month subsequently, his symptoms had resolved and the abscess had diminished in size based upon the features of his follow-up assessment computed tomography (CT) scan. He was referred to the clinic of the authors again with obstructive lower urinary tract symptoms and medical treatment was commenced anew. In view of failed medical treatments, open prostatectomy was undertaken 5 months later. The patient was completely 
symptom-free at the time of the publication of the case report. Basiri et al. [42] made the ensuing summations related to prostate abscess:

- It has been documented that prostate abscess is not a common disease which has an incidence rate of $0.5 \%$. (3) [6]

- The commonest manifesting symptoms of prostate abscess include: dysuria, urinary frequency, perineal pain, fever, chills, as well as low back pain. [43] [44]

- $\quad$ Prostate abscess mostly occurs in the 5th and 6th decades of life and it has been stated that the most common organism that tends to be cultured from prostate abscess pus is Staphylococcus aureus [6] [45] [46].

- It has been iterated that trans-rectal ultrasound scan is the most common diagnostic radiology imaging investigation that is utilized for the diagnosis of the prostate abscess and trans-rectal ultrasound scan is considered to be a good guide for aspiration of the prostate abscess, percutaneous drainage of the prostate abscess, as well as for the assessment of the response to the treatment provided. [11] [45] [47] [48].

- It has been reported in a study on 9 patients who had prostate abscess who had undergone perineal drainage of the abscess and catheter insertion to remove the discharge without irrigation, two patients did experience the recurrence of the abscess in 1-month follow-up and they underwent antibiotic treatment as well as drainage of the abscess again [6].

- In another study of prostate abscess, 6 patients who had prostate abscess underwent perineal aspiration with utilisation of TRUS, and one patient had experienced recurrence of the prostate abscess, who underwent transurethral resection of the prostate. [47]

- With regard to their patient, drainage of the prostate abscess was undertaken transperineally under local anaesthesia with the guidance of TRUS utilising a stent for 5 days and washing with normal saline and antibiotics. In the 3-year follow-up with computed tomography (CT) and trans-rectal ultrasound (TRUS) scan, no recurrence was observed. Hence, this method could be proposed as a less invasive and less morbid method for the treatment of the prostate abscess. Nevertheless, further studies on the management of prostate abscess would be necessitated.

El-Shazly et al. [49] stated that the incidence of prostate abscess (PA) has markedly decreased with the widespread utilization of antibiotics and the decreasing incidence of urethral gonococcal infections. El-Shazly et al. [50] evaluated different treatment options for prostate abscess in order to describe technical points which would improve the outcome of transurethral (TUR) drainage of prostate abscess. El-Shazly et al. [49] undertook a retrospective study of a series of 11 patients who were diagnosed as having prostate abscess, who had been admitted and treated within Farwaniya Hospital, Kuwait, between February 2008 and November 2010. Drainage of the prostate was indicated when antibiotic treatment was not ensued by clinical improvement and after the diagnosis of prostate abscess was confirmed by TRUS (Transrectal ultrasonography) and/or CT computed Tomography scan. TUR drainage was undertaken in 7 cases, ultrasound-guided trans-rectal drainage was undertaken in 2 cases, and ultrasound-guided perineal drainage was undertaken in 2 cases. With regard to the results, El-Shazly et al. [49] reported that all of the patients had undergone Trans-urethral-drainage (TUR-drainage) of the prostate and they all had successful outcomes without the need to undergo secondary treatment or further surgery. ElShazly et al. [49] made the ensuing conclusions:
- $\quad$ Trans-urethral resection (TUR) drainage of a prostate abscess does increase the likelihood of a successful outcome and does lower the incidence of treatment failure or repeated surgery.

- Less invasive treatment, with perineal or transrectal aspiration, might be preferred as a primary treatment in relatively young patients who have localized prostate abscess cavities.

Granados et al. [6] reported a series of 25 men who had prostate abscesses that they had studied during an 11-year interval in whom prostate fluctuation upon digital rectal examination was the most characteristic sign. Transrectal ultrasound was the most reliable method for diagnosis and transurethral drainage under antibiotic coverage was the ideal treatment for this disease.

Baral et al. [50] stated that tuberculosis of the prostate gland is one of the rarest findings within clinical practice and abscess of prostate associated with tuberculosis of the prostate gland is even scarce in the reported literature. Baral et al. [50] reported a case of tuberculosis of the prostate gland which was complicated by huge prostate abscess. Baral et al. [50] reported a 68-year-old man who did not have any comorbidity who had manifested with a history of increased frequency of voiding that was associated with poor urinary flow, urinary urgency as well as nocturia over the preceding 17 days. He had been undergoing medical treatment for benign enlargement of his prostate gland for a period of 2 years. He had digital rectal examination which demonstrated a boggy cystic swelling anteriorly and enlarged prostate gland with mild tenderness. He had ultrasound scan of his abdomen and pelvis which demonstrated massive enlargement of his prostate gland with central avascular necrotic area with moving internal echoes. He had contrast-enhanced computed tomography (CECT) scan which demonstrated a 230 grams of prostate gland with central liquefaction of about 101 cubic mm3. He underwent trans-urethral loop drainage of the prostatic abscess together with transurethral resection of the prostate gland (TURP). Histopathology examination of the specimen demonstrated granulomatous prostatitis which was highly suggestive tuberculosis of the prostate gland. His prostatic abscess culture was negative. At the time of the case report, the patient was undergoing category 1 anti-tuberculosis treatment.. Baral et al. [50] stated the following:

$\checkmark \quad$ Tuberculosis of the prostate gland is an uncommon clinical finding which tends to be commonly encountered disseminated tuberculosis in individuals who have $\mathrm{u}=$ immunocompromised status.

$\checkmark$ Abscess of the prostate gland within the setting of granulomatous tuberculosis of the prostate gland is even rare.

$\checkmark \quad$ Trans-rectal ultrasound scan of the prostate gland tends to be the investigation of choice of prostatic abscess if the ultrasound scan facility is available.

$\checkmark \quad$ The treatment of tuberculosis prostatic abscess does tend to include drainage of the abscess preferably by means of transurethral drainage of the abscess with resection of the prostate and anti-tuberculosis treatment.

.Baral et al. [50] concluded that trans-urethral loop drainage of prostatic abscess is a safe treatment option for patients who manifest with tuberculous prostatic abscess which is then followed by anti-tuberculosis treatment if the histopathology examination features of the specimen are suggestive of granulomatous lesions of the prostate gland.

Kim et al. [51] stated that Klebsiella pneumonia (KP) is related to a metastatic phenomenon from the originally affected primary organ and that approximately $28 \%$ of patients who have pyogenic liver abscess arising from KP do suffer from metastatic complications. Kim et al. [51] undertook a study I order to define the clinical features of KP-induced 
prostatic abscess. Kim et al. [51] reported that a total of 14 patients were diagnosed as having prostate abscess based upon clinical, laboratory examination and abdominopelvic computed tomography (CT) scan from 2007 to 2013. Kim et al. [51] summarised the results as follows: Out of the 14 patients, KP was the dominant causative microorganism in 6 patients that amounted to $42.9 \%$ of the patients, which was followed by Escherichia coli in 2 patient, Pseudomonas aeroginosa in 1 patient, methicillin-resistant Staphyolcoccus aureus in 1 patient, and no growth in either the urine or blood culture in 4 patients. Four patients that amounted to $66.7 \%$ of the $6 \mathrm{KP}$ induced-prostate abscess had other concurrent abscess sites besides the prostate that included the liver in 3 patients, the kidney in 1 patient, and the perianal area with endogenous endophthalmitis that ended in loss of vision in 1 patient. Kim et al. [51] made the following conclusions:

- They had reported on the clinical features of KP-induced prostate abscess based upon a small number of patients, which does represent the main limitation of their study.

- It was their belief that if the causative organism of a prostate abscess was KP, more workup would be needed to exclude the presence of an abscess in other organs, especially within the liver.
- $\quad$ Abdominopelvic computed tomography (CT) scan would be a proper radiology imaging modality.

Ullah et al. [52] reported a 60-year-old man who manifested with fever, chills, cough, and shortness of breath. He denied having any abdominal pain, urinary urgency, dysuria, or haematuria. The results of his routine haematology and biochemistry laboratory tests demonstrated an elevated white blood cell count and lactic acid, and one set of blood culture stained positively for gram-positive cocci. He was empirically commenced on intravenous antibiotics. On the next hospital day, the patient complained of haematuria and lower abdominal discomfort. He had computed tomography scan of his abdomen and pelvis which demonstrated focal hypodensity relating to his prostate abscess, which was subsequently confirmed based upon magnetic resonance imaging (MRI) scan of the prostate (see figures 1 and 2). The final report of his blood cultures confirmed a growth of methicillin-resistant Staphylococcus aureus. He was treated by means of intravenous vancomycin and transurethral resection as well as deroofing of the prostate gland with drainage of the abscess fluid which resulted in complete resolution of his symptoms. Patient was reviewed within the hospital of the authors by the Urology team about 3 weeks later, and he did not have any urological issues. He had repeated blood and urine cultures which did not report any growth of an organism. He received a total of 1 month of antibiotic treatment

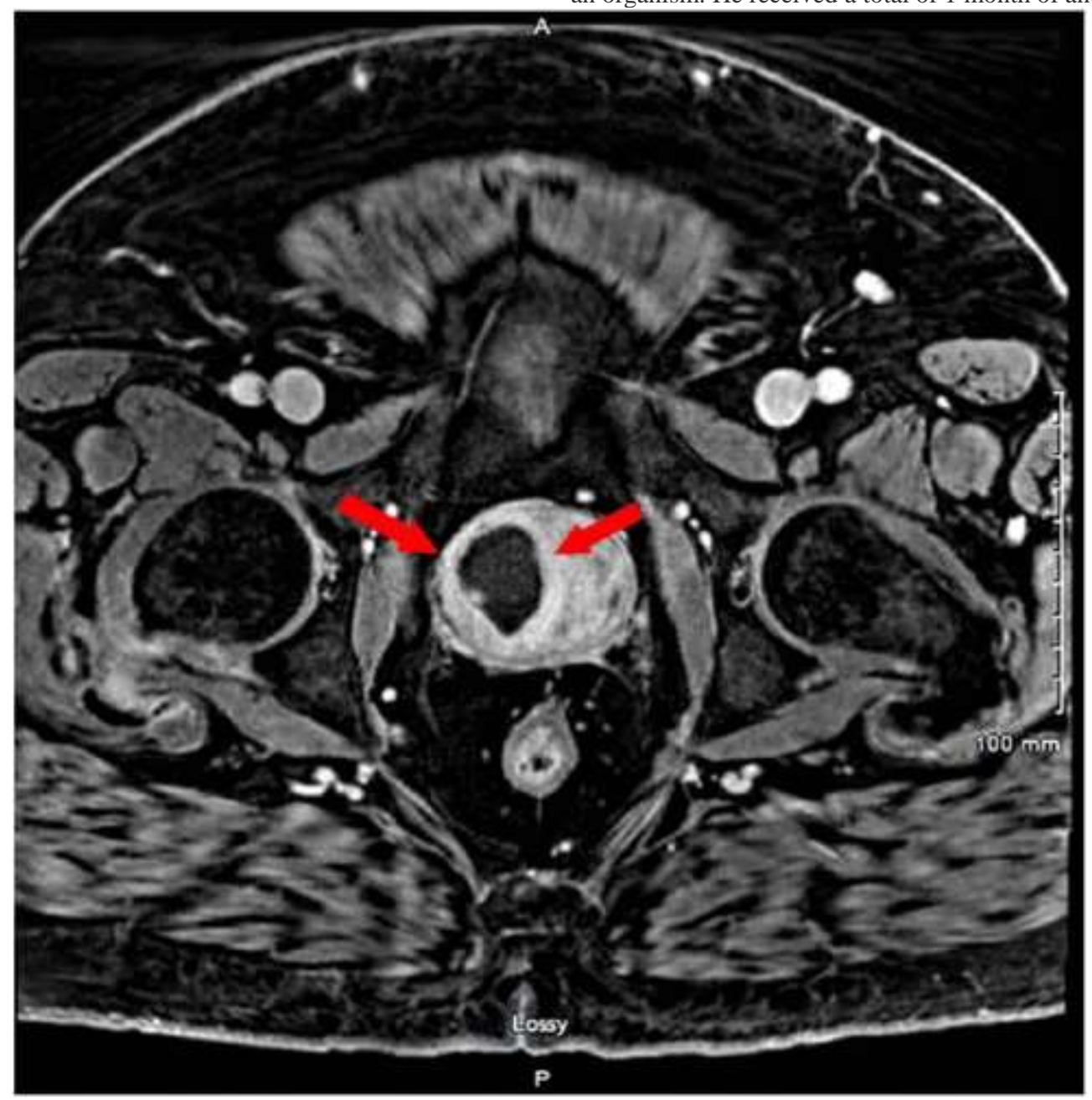

Figure 1: Magnetic resonance imaging axial view showing cystic lesion (red arrows) within the right aspect of prostate gland with marked restriction of diffusion. Reproduced from [52]: This article is distributed under the terms of the Creative Commons Attribution 4.0 License (http://www.creativecommons.org/licenses/by/4.0/) which permits any use, reproduction and distribution of the work without further permission provided the original work is attributed as specified on the SAGE and Open Access pages (https://us.sagepub.com/en-us/nam/open-access-at-sage). 


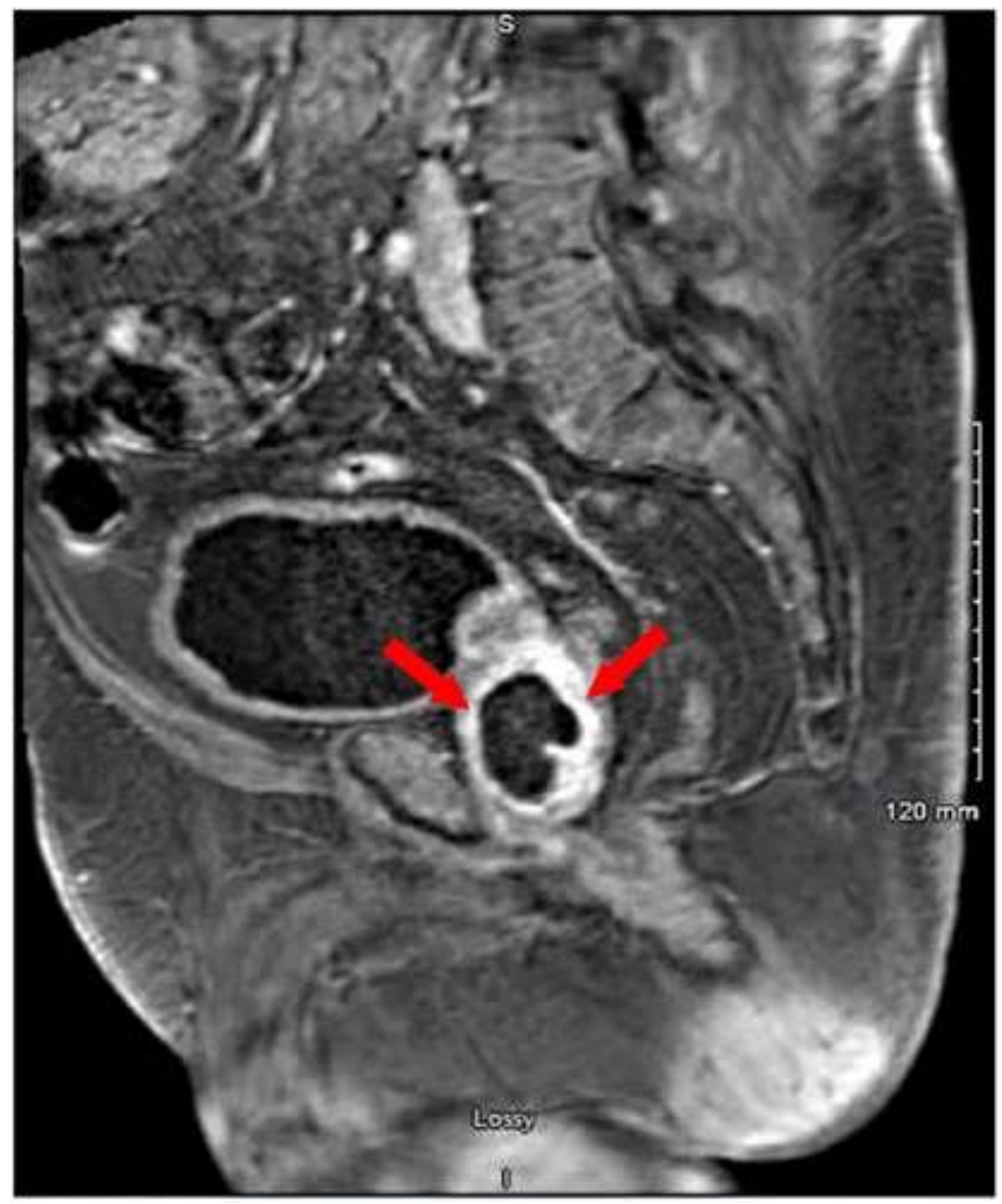

Figure 2: Magnetic resonance imaging sagittal view showing rim enhancing prostatic lesion (red arrows). Reproduced from [52]:This article is distributed under the terms of the Creative Commons Attribution 4.0 License (http://www.creativecommons.org/licenses/by/4.0/) which permits any use, reproduction and distribution of the work without further permission provided the original work is attributed as specified on the SAGE and Open Access pages (https://us.sagepub.com/en-us/nam/open-access-at-sage).

Deshpande et al. [53] made the ensuing iterations about prostate abscess:

- Prostatic abscess is an uncommon complication of an ascending urinary tract infection (UTI).

- The incidence of prostate abscess incidence has reduced secondary to routine and early utilization of antibiotics for the treatment of UTIs.

- Prostate abscess had been reported in patients who have uncontrolled diabetes mellitus, prolonged indwelling urinary catheters, prostate biopsy or other instrumentation of lower urinary tract.

- $\quad$ Prostate abscess most commonly tends to be associated with Gram-negative bacteria.

- Staphylococcus aureus is rarely implicated in prostate abscess and it has been reported in patients with underlying risk factors like long-term or uncontrolled diabetes, intravenous drug abuse or bacteraemia.
- They had reported a rare case of prostate abscess due to methicillin resistant Staphylococcus aureus without obvious risk factors.

Deshpande et al. [53] reported a 49-year-old man who manifested with 5 and tenderness of the scrotum and adjacent perineum. He had an elevated white blood cell count and his blood and urine days of fever and chills that was associated with scrotal pain and swelling. He was found upon examination to have a temperature of 100.7 degrees Fahrenheit cultures were negative. He had contrast-enhanced computed tomography (CT) scan which demonstrated an enlarged prostate gland which contained a 4 $\mathrm{cm}$ septated hypodense lesion that had contrast-enhancing walls as well as encompassing fatty inflammation. He was commenced upon ciprofloxacin antibiotic treatment. He underwent cystoscopy-guided trans-urethral drainage of the abscess together with resection of the prostate gland with removal of about $50 \mathrm{ml}$ of purulent pus. Culture of the pus grew MRSA that was sensitive to vancomycin, tetracycline, ciprofloxacin, as well as trimethoprim/sulfamethoxazole. Histopathology examination of the resected specimens demonstrated benign prostate tissue and urothelial mucosa with evidence of acute and chronic inflammation. His antibiotic treatment was changed to intravenous 
vancomycin. He discharged himself against medical advice but he was discharged home on oral doxycycline which he continued for 6 weeks. He had a follow-up CT scan 8 weeks pursuant to his initial manifestation which demonstrated marked interval improvement.

Sakamati et al. [54] sated that Nocardia farcinica usually does infect the respiratory tract and it can sometimes cause central nervous system infections; nevertheless, Nocardia farcinia rarely infects the prostate gland. Sakamaki et al. [54] reported the first case of N. farcinica that was identified within the purulence specimen that was drained from a prostate abscess. A 70-year-old Japanese man who was receiving steroid and cyclosporine treatment presented with chief complaint of turbid urine. His urine microscopy demonstrated 100 white blood cells per high power field and bacteria. He had computed tomography (CT) scan of abdomen and pelvis which showed a low-density lesion within his prostate gland that measured $4.0 \mathrm{~cm} \times 5.0 \mathrm{~cm} \times 5.0 \mathrm{~cm}$ (see figure 3). Gram staining of his urine did demonstrate gram-positive, filamentous, branching rods (see figure 4a). Kinyoun staining of his urine demonstrated filamentous, branching rods (see figure $4 \mathrm{~b}$ ). His urine culture was positive for $\mathrm{N}$. farcinica, and the presence of this organism was confirmed by mass spectrometric analysis. The antibiotic drug sensitivity analysis of the cultured organism is demonstrated in Table 1.

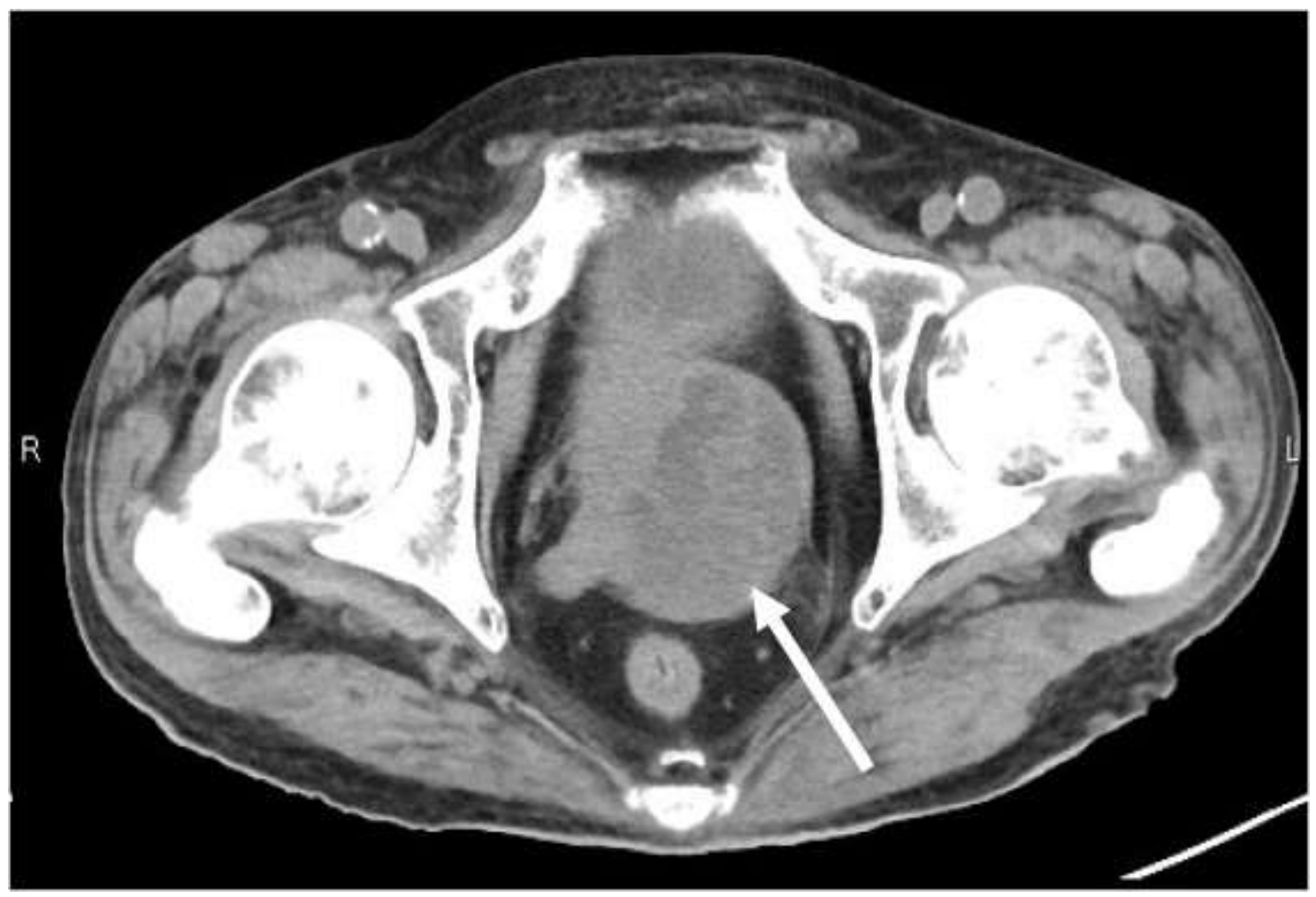

Figure 3: CT scan: A pelvis CT revealed a $4.0 \times 5.0 \times 5.0 \mathrm{~cm}$ low density lesion indicate abscess at left side of prostate. Reproduced from: [54]
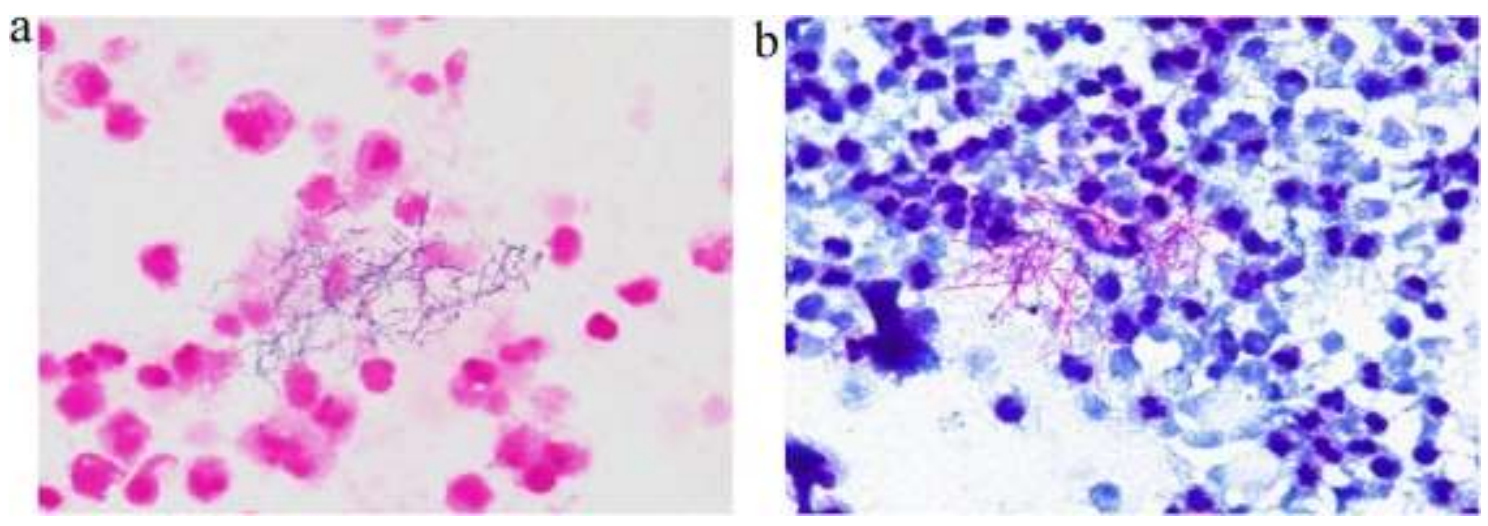

Figure 4 a: Gram stain: Urine Gram stain showed filamentous branching Gram-positive rods and white blood cell $(\times 1000)$. b. Kinyoun stain: Urine Kinyoun stain showed filamentous branching rods $(\times 1000)$. Reproduced from: [54] 


\begin{tabular}{|l|c|c|}
\hline Antibiotics & MIC $(\boldsymbol{\mu g} / \mathbf{m L})$ & Interpretation \\
\hline CVA/AMPC & 4.0 & $\mathrm{~S}$ \\
\hline CTRX & 16.0 & $\mathrm{I}$ \\
\hline IPM/CS & 2.0 & $\mathrm{~S}$ \\
\hline CAM & 8.0 & $\mathrm{I}$ \\
\hline MINO & 4.0 & $\mathrm{I}$ \\
\hline CPFX & 8.0 & $\mathrm{R}$ \\
\hline TOB & 8.0 & $\mathrm{I}$ \\
\hline AMK & 0.5 & $\mathrm{~S}$ \\
\hline TMP/SMX & 0.25 & $\mathrm{~S}$ \\
\hline
\end{tabular}

S: Susceptible, I: Intermediate, R: Resistant.

CVA/AMPC: Amoxicillin/Clavulanate, CTRX: Ceftriaxone, IPM/CS: Imipenem/Cilastatin.

CAM: Clarithromycin, Minocycline, CPFX: Ciprofloxacin, TOB: Tobramycin.

AMK: Amikacin, TMP/SMX: Trimethoprim/Sulfamethoxazole. Reproduced from [54]

Table 1: Drug susceptibility of N. farcinica identified our patient.

He received treatment by means of oral trimethoprim/sulfamethoxazole (TMP/SMX) $320 / 1600 \mathrm{mg} /$ day $(6.4 / 32 \mathrm{mg} / \mathrm{kg} / \mathrm{day})$. On the $6^{\text {th }}$ day pursuant to commencement of his treatment, his serum creatinine level was raised at $1.53 \mathrm{mg} / \mathrm{dL}$. Because of his acute kidney injury, his antimicrobial treatment was changed to imipenem/cilastatin (IPM/CS) $1.5 \mathrm{~g} /$ day $(30 \mathrm{mg} / \mathrm{kg} / \mathrm{day})$. On the $11^{\text {th }}$ day from the commencement of his treatment with TMP/SMX, trans-perineal prostatic drainage with percutaneous vesicostomy was undertaken. The culture of the drained pus was positive for $\mathrm{N}$. farcinica. On the $18^{\text {th }}$ day, the patient was recommenced on oral TMP/SMX $80 / 400 \mathrm{mg} /$ day $(1.6 / 8 \mathrm{mg} / \mathrm{kg} /$ day $)$ that was increased gradually to $320 / 1600 \mathrm{mg} /$ day following discontinuation of the intravenous IPM/CS administration. Nevertheless, his serum creatinine level was found to be raised again. The TMP/SMX dose was reduced to $160 / 800 \mathrm{mg} /$ day $(3.2 / 16 \mathrm{mg} / \mathrm{kg} /$ day $), \quad$ and clavulanate/amoxicillin (CVA/AMPC) $1500 \mathrm{mg} /$ day $(30 \mathrm{mg} / \mathrm{kg} /$ day) was added. After 60 days from the commencement treatment against nocardiosis, the patient was eventually discharged on TMX/SMX $160 / 800 \mathrm{mg} /$ day and CVA/AMPC $2250 \mathrm{mg} /$ day $(45 \mathrm{mg} / \mathrm{kg} /$ day $) . \mathrm{He}$ continued to take the medications for about 12 months in the outpatient setting. Sakamaki et al. [54] made the following conclusions:

- Antibiotic administration and prostatic drainage were effective.

- $\quad$ N. farcinica was detected in the cultures of urine and prostatic drainage purulent specimens.

- Nocardiosis should be included in the differential diagnosis in immunosuppressive patients who have prostate abscess.

Scorey and Daniel. [55] stated the following:

- Nocardia is characterised as a Gram positive filamentous rod and Nocardia is found globally within the soil, decaying vegetable matter and aquatic environments.
- Localised pulmonary infection is the commonest clinical manifestation.

- Nevertheless, Nocardia could manifest in a wide variety of clinical presentations, especially with regard to immunocompromised individuals.

Scorey and Daniel. [55] reported what in their opinion was the first case of a prostate abscess that was caused by Nocardia farcinica in a man who had a history of severe psoriasis and psoriatic arthritis. The man had been on long term immunosuppression for his psoriasis and psoriatic arthritis with prednisolone and etanercept. Scorey and Daniel. [55] felt that the Nocardia was likely contracted via direct skin inoculation while the patient was gardening with haematological dissemination to the prostate. He did respond well to long term sulfamethoxazole and trimethoprim.

Scorey and Daniel. [55] reported a 68 year old man who had manifested in March 2015 with a one week history of intermittent dysuria as well as suprapubic pain. He had a two day history of hesitancy before voiding, fever, vomiting, diarrhoea and dyspnoea. Three weeks prior to his presentation he had cut his left middle finger when he was gardening and which became infected and for which he received a one week course of amoxicillin.

He had been immunosuppressed heavily for 30 years for severe psoriasis and psoriatic arthritis. He had been taking prednisolone $10 \mathrm{mg}$ daily and etanercept $50 \mathrm{mg}$ weekly for the preceding 5 years. Two days into his admission he developed retention of urine for which he was catheterised. He had a digital rectal examination which demonstrated a tender prostate gland. He had computed tomography (CT) scan of abdomen and pelvis which demonstrated a $3.6 \mathrm{~cm} \times 3 \mathrm{~cm} \times 3.4 \mathrm{~cm}$ left prostate abscess as well as a possible sub-centimetre abscess which had involved his right lobe of prostate (see figure 5). His antibiotics were next changed to $500 \mathrm{mg}$ twice per day of intravenous ciprofloxacin. 


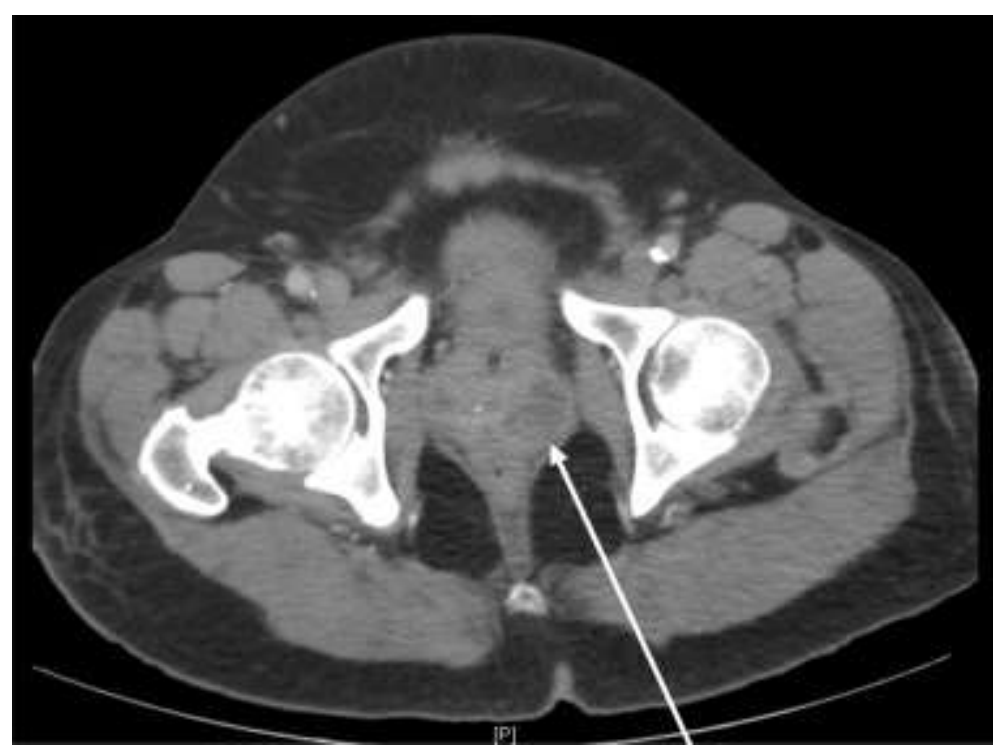

Figure 5: CT Scan with the white arrow pointing to the prostate abscess. Reproduced from [55] under copyright Copyright (0) 2016 The Author(s). This is an open access article under the CC BY-NC-ND license (http://creativecommons.org/licenses/by-nc-nd/4.0/).

On the 6th day his blood culture specimens that were taken prior to commencement of his antibiotics on the first grew N. farcinica. The identification was undertaken by $16 \mathrm{~s}$ rRNA gene sequencing. Trimethoprim and sulfamethoxazole (TMP-SMX) treatment were next started in addition. He furthermore had blood cultures taken on the 7th day as well as 10th day which did not grow any organism. He had a computed tomography (CT) scan of his thorax and brain which did not demonstrate any evidence of Nocardia seeding even though a $5 \mathrm{~mm}$ soft tissue nodule was demonstrated within his right lower lobe. He did respond to treatment and the levels of his inflammatory markers returned to within normal range. He was subsequently discharged home pursuant to 15 days of him being on oral ciprofloxacin and TMP-SMX. The etanercept was withheld indefinitely and prednisolone was weaned back onto his normal dose of treatment. He had at the time of publication of the article been on antibiotics for 8 months and he had had a repeat CT scan which demonstrated resolution of his prostate abscess. Scorey and Daniel. [55] Made the following conclusions:

- Nocardia infection has had a rising incidence in view of the increasing numbers of immunosuppressive patients as well as because of improved methods for the detection of Nocardia infection.

- Early detection and treatment of patients who have Nocardia infection is important clinically in view of its associated high mortality.

- Their reported case does serve to highlight the importance of early, pre-antibiotic blood cultures as well as the need for clinicians to take into consideration unusual manifestations of opportunistic infections with regard to immunocompromised patients.

Goyal et al. [56] reported their experience of prostate abscess management by the undertaking of modified transurethral resection (TUR) technique. With regard to the methods, Goyal et al. [56] reported that Seventeen men who had prostate abscess and had undergone TUR between 2003 and 2011 were retrospectively analysed. Goyal et al. [56] noted the details of demography, surgical procedures, complications, and follow-up. Goyal et al. [56] summarized the results as follows:

- The mean age of the patients was $61.53 \pm 8.58$ years, and all of the patients had multifocal abscess cavities.
- Initially, 6 men did undergo classical TUR that was similar to the technique that is used for benign prostatic enlargement which constituted group 1 .

- Next, 11 men did undergo a modified TUR that constituted group 2 in which the neck of the urinary bladder and anterior zone were not resected.

- The abscess cavities did resolve completely, and none of the patients required a second intervention.

- $\quad$ One patient within group 1 and three patients within group 2 developed post-operative fever that required administration of parenteral antibiotics $(\mathrm{P}=0.916)$.

- Three patients within group 1 developed transient urinary incontinence; nevertheless, none of the patients within group 2 developed this complication $(\mathrm{P}=0.055)$.

- Four and five men within group 1 and 2 did report retrograde ejaculation, respectively $(\mathrm{P}=0.740)$.

Goyal et al. [56] made the following conclusions:

- The modified technique of prostate resection does have an edge over the conventional TURP in the form of reduced morbidity but it does maintain its high success rate for complete drainage of abscess.

- The modified technique of prostate resection, does alleviate the need for the undertaking of secondary procedures, and it does have an apparent advantage over limited drainage techniques.

- They would recommend the undertaking of this technique which is emphasized in cases that are associated with benign prostatic hyperplasia (BPH) and lack of proper preoperative imaging.

Gilmore et al. [57] reported a healthy 6-year-old man who had rare case of a prostate abscess which he had developed following iodine-125 seed brachytherapy he had undergone as treatment for prostate adenocarcinoma. Gilmore et al. [57] iterated that the only other documented case of prostate abscess that developed after brachytherapy was reported in an HIV-positive man whose underlying pathology turned out to be squamous cell carcinoma of the prostate and rectum. 
Bae et al. [58] stated that emphysematous prostatic abscess is a very uncommon form of prostatitis and that emphysematous prostate abscess due to Klebsiella pneumoniae could have a poor prognosis according to a few previous reports. Bae et al. [58] reported a rare case of successfully treated emphysematous prostate abscess with cystitis due to Klebsiella pneumoniae in a 50-yr-old man who had a 15-year history of diabetes mellitus, who was referred to the emergency room of their hospital. The KUB film demonstrated gas shadows within the lower pelvic area which was suggestive of emphysematous cystitis or emphysematous prostatic abscess. The gas had mainly occupied the prostate and was also observed in the bladder on computed tomography (CT) scan of pelvis. He was successfully treated with long-term antibiotic utilisation and additional percutaneous drainage of the abscess. Bae et al. [58] made the ensuing iterations:

- Emphysematous prostatic abscess could be misdiagnosed as emphysematous cystitis in view of the similar location of gas shadows on radiography.

- Computerized tomography (CT) scan and transrectal ultrasonography are helpful with regard to making the diagnosis of emphysematous prostatic abscess.

- Appropriate utilization of effective antibiotics with drainage of pus is the best treatment.

- $\quad$ Their reported case does emphasize the importance of timely and accurate diagnosis followed by appropriate treatment in emphysematous prostatic abscess in diabetic patients.

Share this article Share with email Share with twitter Share with linkedin Share with facebook

Chuang et al. [59] reported a young alcoholic and heavy smoker who was admitted because of Klebsiella pneumoniae bacteraemia. He had Contrast-enhanced Computed tomography (CT) scan of abdomen and pelvis which demonstrated an abscess within his the right lobe of his prostate gland. He did develop blurred vision of his left eye on the third day during his admission. His initial eye examination revealed mild reaction within his anterior chamber and vitreous as well as multiple Roth's spots within his retina. The administration of empirical antibiotics that included cefazolin and gentamicin intravenously was changed to ceftriaxone and amikacin following ophthalmologic consultation. After he had received parenteral antibiotics for 3 weeks, his systemic condition was controlled, and his vision did remain stable. Chuang et al. [59] stated that over the past decades, many cases of endogenous Klebsiella pneumoniae endophthalmitis that were associated with liver abscess had been reported in Taiwan. Nevertheless, documented reports of this condition associated with a prostate abscess were not common but rare. Chuang et al. [59] reported a case of Endogenous Klebsiella pneumoniae endophthalmitis that was associated with prostate abscess:, which was classified as posterior focal endogenous endophthalmitis, that had good prognosis under appropriate antibiotic treatment. Chuang et al. [59] iterated that early diagnosis and prompt treatment are important for the control of sepsis and restoration of vision.

Stafford and Prybys. [60] reported a 57-year-old African American male patient who had a past history of hypertension and long-standing endstage renal disease (ESRD) who was on maintenance haemodialysis through a left upper extremity arteriovenous fistula who had manifested with penile pain and discharge for one day. He did report that he had been anuric for many years; nevertheless, on the day preceding his presentation, he had started experiencing a scanty amount of thick urethral discharge. His genitourinary examination demonstrated a scanty amount of urethral discharge and no other abnormality. He had a right-sided inguinal lymphadenopathy. His digital rectal examination demonstrated an enlarged and boggy as well as tender prostate gland upon palpation. A urethral swab of his discharge fluid was obtained for gram stain, culture, and polymerase chain reaction assay for gonorrhoea and chlamydia DNA. Resistance to swab passage within the distal urethra was noted. In view of concern for him having prostatitis and sexually transmitted infection, he was given a single-dose treatment regimen of ceftriaxone, azithromycin, and metronidazole, with a plan to prescribe for him ciprofloxacin as outpatient treatment of prostatitis. Nevertheless, about 60 minutes after the urethral swabbing was undertaken, he then developed a large amount of purulent drainage from his urethra, which was cultured. His repeat vital signs at that time showed a heart rate of 120 , blood pressure 160/100, respiratory rate 22, and temperature 101.7 Fahrenheit which established a diagnosis of early sepsis, which was initially considered to be secondary to pyelonephritis in the setting of anuria. Following various routine laboratory investigations and blood culture specimens being sent to the laboratory he was commenced on vancomycin and piperacillin/tazobactam. He had contrast-enhanced computed tomography (CECT) of the abdomen and pelvis which demonstrated communication between the posterior bladder wall and the seminal vesicles and a large prostatic abscess (see figure 6). Following his admission to the hospital, the a Foley urethral catheter was inserted for further bladder irrigation and drainage. Following a successful drainage of the prostate abscess through a percutaneous drain that was inserted by an interventional radiologist, the Foley catheter was removed.

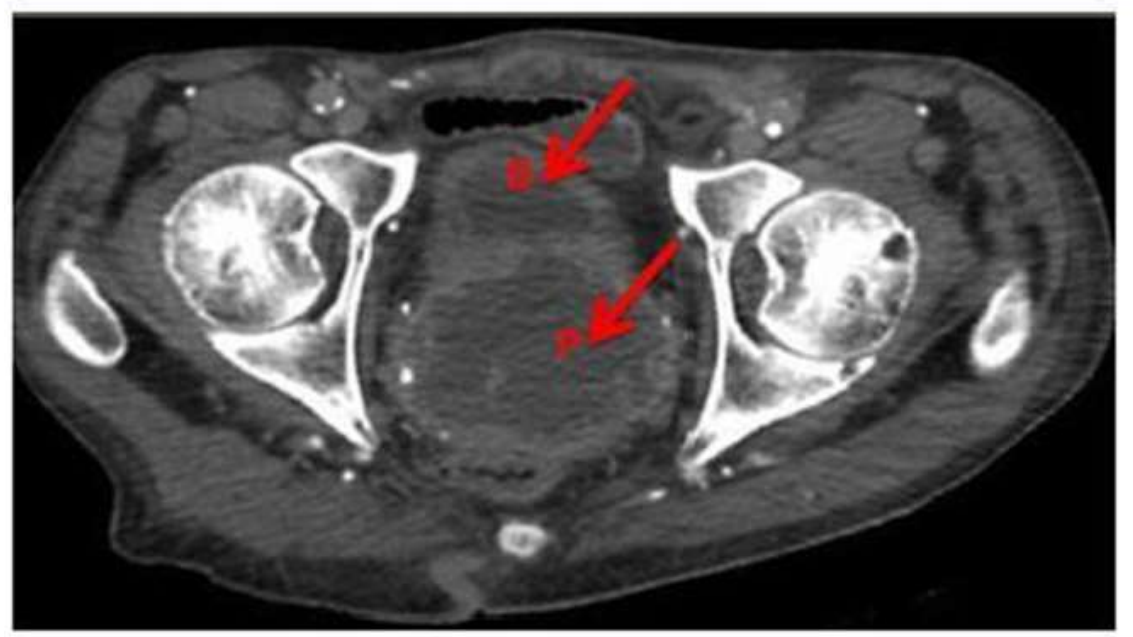

Figure 6: Axial computed tomography demonstrates persistent abscess within the bladder and prostate after spontaneous urethral drainage, with communication between the bladder $(B)$ and prostate $(P)$ abscesses. Reproduced from: [60] 
The results of cultures of the discharge fluid and of the prostate abscess after the drain was inserted both demonstrated Escherichia coli which was resistant to ampicillin, gentamicin, tetracycline, tobramycin, and trimethoprim/sulfamethoxazole, and sensitive to cephalosporins, carbapenems, fluoroquinolones, and piperacillin/tazobactam. His blood and stool cultures did not grow any organism. He recovered and was discharged on a two-week course of ciprofloxacin with urology follow up.

Liao et al. [37] stated the following:

- Prostate abscess usually tends to be a complication of acute urinary tract infection.

- Invasive liver abscess syndrome is characterized with Klebsiella pneumoniae related multiple organ metastasis.

- Concomitant pyogenic liver abscess and prostate abscess had rarely been reported.

- Recurrent episode of liver abscess also tends to be even rarer.

Liao et al. [37] reported a 71-year-old man who had acute bacterial prostate abscess and urinary tract infection that was caused by Klebsiella pneumoniae that was associated with multiple liver abscess, psoas muscle abscess and osteomyelitis. His blood culture and urine culture grew Klebsiella. pneumoniae, which confirmed the diagnosis of invasive liver abscess syndrome that was caused by Klebsiella pneumoniae. $\mathrm{He}$ was successfully treated with empirical antibiotics for 6 weeks. Liao et al. [37] made the following conclusions:

- $\quad$ Their reported case had emphasized the importance of timely and accurate diagnosis which should be followed by appropriate treatment in disseminated Klebsiella pneumoniae infection in order to prevent the development of morbidity as well as mortality.

- In a patient who has impaired host defences such as diabetes mellitus or alcoholism related liver cirrhosis, clinicians should always keep invasive liver abscess syndrome in mind.

- Once this syndrome is confirmed, various radiology imaging studies for the metastatic lesion, utilization of empirical antibiotics and early adequate drainage of the liver abscess should be undertaken as soon as possible.

Walker et al. [61] stated the following:

- Prostate abscess (PA) is an uncommon infection which is generally secondary to Escherichia coli and other members of the Enterobacteriaceae family.

- In recent years, even though uncommon, more reports of Staphylococcus aureus (S. aureus) PA have been reported, especially with increasing reports of bacteraemia that has been associated with injection drug use (IDU).

Walker et al. [61] undertook a retrospective review of adult patients who were admitted to a tertiary care hospital between 2008 and 2018 and who had a diagnosis of Staphylococcus. aureus Prostate Abscess. Walker et al. [61] summarised the results as follows:

- Twenty-one patients were included in the study.

- The average age of the patients was 46 years.

- Fourteen that amounted to $67 \%$ of the patients had manifested with genitourinary concerns.
- The main risk factors for the development of prostate abscess (PA) included concurrent skin or soft tissue infections in 52\% of the patients, a history of genitourinary disease or instrumentation in $48 \%$ of the patients, IDU in $38 \%$ of the patients, and diabetes mellitus in $38 \%$ of the patients.

- Methicillin-resistant Staphylococcus aureus (MRSA) was identified in $57 \%$ of the patients and concomitant bacteraemia occurred in $81 \%$ of the patients.

- Surgical or a radiologically guided drainage of the prostate was undertaken in $81 \%$ of the patients.

- The duration of antibiotic treatment did range from 3 weeks to 8 weeks.

- Six patients were lost to follow-up.

- Clinical resolution was occurred in the remaining 15 patients that amounted to $81 \%$ of the patients who had follow-up.

Walker et al. [61] made the following conclusions:

- Staphylococcus aureus (PA) has continued to be a rare complication of Staphylococcus aureus infections.

- In majority of published reports, Methicillin Resistant Staphylococcus Aureus (MRSA) has been the culprit.

- In high risk patients who have persistent bacteraemia, physicians do need to consider the prostate as a site of infection.

Arrabal-Polo et al. [62] stated that the incidence of prostate abscess is $0.5 \%$ in relation to all prostate pathologies and that prostate abscess usually does occur in patients who have diabetes mellitus or with some degree of immunosuppression. Arrabal-Polo et al. [62] reported the case of an 84 years-old man who had a history of arterial hypertension and mild renal failure, who had manifested with high fever, prostate syndrome, genital oedema and constipation. He was diagnosed as having prostate abscess by means of trans-rectal ultrasonography (TRUS). His treatment was commenced with empirical meropenem and a puncture of the abscess was undertaken transperineally under TRUS guidance with insertion of an 8-Fr nephrostomy tube for 36 hours. He was discharged 48 hours pursuant to the puncture of the prostate abscess with a good prognosis. Arrabal-Polo et al. [62] made the following conclusions:

- $\quad$ TRUS-guided trans-perineal drainage is a safe, adequate as well as effective treatment for prostate abscess, and it does allow the placement of drainage for several hours and thereby it does avoid communication between the abscessed cavity and the urethra or the rectum.

- In view of this, after having reviewed the literature, they consider this approach suitable for drainage of prostate abscess.

Quraishi et al. [63] stated the following:

- Staghorn calculus is a calculus that does accommodate the majority of a renal calyx extending into the renal pelvis.

- A conservative approach to the treatment of Staghorn calculus could lead to high morbidity and mortality rates.

- Such morbidity usually tends to present with renal failure, obstructed upper urinary tract as well as / or life-threatening sepsis.

- $\quad$ Prostate abscesses had previously never been associated with staghorn calculi in the literature. 
Quraishi et al. [63] reported a case of a 70-year-old man who had manifested with sepsis, that was found to have originated from a complex prostate abscess. The patient did not have any history of urinary tract infections or risk factors. Quraishi et al. [63] believed that the incidentally identified staghorn calculi had promoted the growth of Proteus mirabilis which had led to the development of the prostate abscess. The patient underwent a transurethral resection of his prostate gland and drainage of the abscess following a failed course of antibiotic treatment. Quraishi et al. [] stated that their reported case also highlighted the paucity of guidelines that were available for the treatment of prostate abscesses.

Dakdouki et al. [64] stated that Brucellosis is a multi-system disease which tends to be endemic in many Mediterranean countries including Lebanon. Nevertheless, human Brucella prostate abscess had not been previously reported in the English medical literature. Dakdouki et al. [64] reported the case of a young man who had manifested with fever and urinary symptoms, and who subsequently was demonstrated to have culture confirmed Brucella prostate abscess. The culture of drained prostate pus grew Brucella melitensis.

Alenazi et al. [65] reported a 54-year-old man, who was a known diabetic patient and who had manifested with a chief complaint of dysuria, weak urine stream, suprapubic pain radiating to perineal area, urethral pus discharge, and fever for about a 2-week duration. His symptoms had not improved pursuant to receiving a 1 week course of antibiotics. He reported that he had ingested raw milk prior to the commencement of his presenting symptoms. Upon examination, he was found to have highgrade fever otherwise his vital sign examination findings were unremarkable. He had a digital rectal examination which revealed an enlarged and tender prostate gland. The results of his laboratory tests revealed mild leucocytosis and elevated lymphocytes as well as monocytes. His urine analysis demonstrated pus cells as well as red blood cells. He had trans-rectal ultrasonography and computed tomography (CT) scan which did confirm the diagnosis of prostate abscess. Transurethral drainage of the prostate abscess, was undertaken for the patient and foley's and suprapubic catheter were inserted to relieve the obstruction. The culture of his prostatic pus culture and titration were positive for Brucella Bactria. Postoperatively, he received intravenous (IV) fluids and antibiotics which included sulfamethoxazole/trimethoprim and gentamicin. One week following his surgery, he developed low-grade fever and his urine culture grew Staphylococcus species and he received ciprofloxacin. Two weeks later, he had improved and he was discharged home on Sulfamethoxazole/trimethoprim. During his follow-up assessment, CT scan, was undertaken and no further collection of abscess was identified with evidence of complete resolution of the prostate abscess.

Alenazi et al. [65] made the ensuing summing iterations:

Prostatic abscess is uncommon case in clinical practice especially with prevalent use of antibiotics. [66] There are different mechanisms exist regarding causation, which include acute or chronic prostatitis; retrograde stream of contaminated urine; or hematogenous spread of distant infection. [67] Predisposing factors for the incidence of prostatic abscess include diabetes, urinary catheter, bladder outlet blockade, chronic renal failure, and chronic liver disorder. [68] It has been stated that, prostatic abscess is mainly caused by Gram-negative enterobacteriaceae including E. coli (Barozzi et al., 1998). [11] Their patient was known to have diabetes as a predisposing risk factor with history of ingestion of raw milk. The patient was diagnosed as having brucellosis based upon serology test and thus was proposed to be the source for hematogenous spread of the infection into prostate. The obtained pus culture demonstrated that the prostate abscess contained Brucella organisms. The patient was treated by means of intravenous (IV) fluids and antibiotics that included sulfamethoxazole/trimethoprim, Gentamicin, and ciprofloxacin. Transurethral drainage of the prostate abscess was undertaken for the patient with foley's and suprapubic catheter insertion to obviate the obstruction.

Alenazi et al. [65] summated their case report by stating the following:

- $\quad$ They had reported a new case of prostate abscess which was caused by Brucella organisms which had been proven by using pus culture and serology.

- These organisms should be suspected in patients who manifest with clinical picture of prostate abscess especially in endemic areas.

Alenazi et al. [65] made the following conclusions: (a) Their reported case was the first case of prostate abscess that was caused by Brucella bacteria in Saudi Arabia. (b) clinicians should consider Brucella infection in their history and management approach for patients who have clinically suspected prostate abscess.

Gupta et al. [69] stated the following:

- Surgical or radiology image-guided drainage of prostate abscess is the treatment of choice for prostate abscesses, together with utilisation of appropriate antibiotics.

- Fungal prostate abscesses tend not to be common, and amphotericin or azoles tend to be the usual agents that are used for the treatment of fungal prostate abscesses.

- Up to the time of report of their case, echinocandins had not been reported as agents of choice for fungal abscesses in view of a concern related to their large molecular size as well as high protein binding.

Gupta et al. [69] reported a case of Candida glabrata prostate abscess which was successfully treated with utilisation of micafungin. Gupta et al. [69] also reported that adequate concentrations of micafungin were demonstrated during the treatment of the fungal prostate abscess.

Lin and Patel. [25] reported a 50-year-old immunocompetent man who had a past medical history that was significant for childhood malaria who had manifested with fevers and myalgias pursuant to his trip to Ghana one week preceding his presentation. He at first denied any focal infectious symptoms, including cough, chest pain, and shortness of breath, dysuria, nausea, abdominal pain, or diarrhoea. During his trip to Ghana, he stayed in a rural village that had indoor plumbing, and he ate home-cooked food, as well as he drank bottled water, and he was not aware of having had any contact with any individual or individuals who were sick or ill. His vital signs upon presentation were as follows: temperature $102^{\circ} \mathrm{F}$, heart rate $133 \mathrm{bpm}$, blood pressure $127 / 88 \mathrm{mmHg}$, and oxygen saturation $98 \%$ on ambient air. Upon his clinical examination he did appear to be fatigued with dry mucous membranes, he was tachycardic with a regular rhythm, apart from that no other significant findings were found upon his examination. The results of laboratory tests were summarised as follows: White blood cell count of $12,000 / \mu \mathrm{L}$ with $86.1 \%$ neutrophils and 70 bands $/ \mu \mathrm{L}$. The results of his first blood cultures, urinalysis, and chest $\mathrm{X}$ ray were unremarkable. In view of his recent travel and concern for infection, a broad infectious work-up was undertaken to ascertain the cause of his symptoms. The results of his nasal influenza/respiratory syncytial viral swab, HIV-1/HIV-2, dengue IgM, Zika IgM, and blood smears for malaria were reported as negative. Considering his persistent high fevers and recent travel to an endemic area for malaria, he was empirically treated for malaria with atovaquone/proguanil which was not associated with any significant improvement in his symptoms. On the $2^{\text {nd }}$ day of his admission to hospital 2, the patient had a temperature of $101.6^{\circ} \mathrm{F}$ and developed dysuria as well as visible haematuria. He had a repeat urinalysis which showed pyuria and $2+$ blood, and he was empirically commenced on ceftriaxone. The results of his urine culture 
subsequently demonstrated ceftriaxone-sensitive Salmonella non-typhi Group B serotype. He continued to have fevers and on his $4^{\text {th }}$ day of admission to hospital, he did develop non-bloody diarrhoea. He had stool bacterial testing and this demonstrated the same strain of Salmonella. The results of his blood cultures continued to remain negative. In view of his persistent fevers, he had a contrast-enhanced computed tomography (CECT) scan of his abdomen and pelvis which demonstrated multi-focal prostate abscesses (see figure 7). He patient underwent trans-gluteal drainage of his prostate abscesses and the resultant cultures grew the same Salmonella pathogen. His fever curve subsequently improved, and following this, he was discharged on an extended course of trimethoprimsulfamethoxazole. The lesson that needs to be learnt from this case report is that Salmonella prostate abscess though uncommon does exist and for this reason clinicians need to have a high index of suspicion for Salmonella prostate abscess especially in individuals who travel to and back from Salmonella endemic areas.

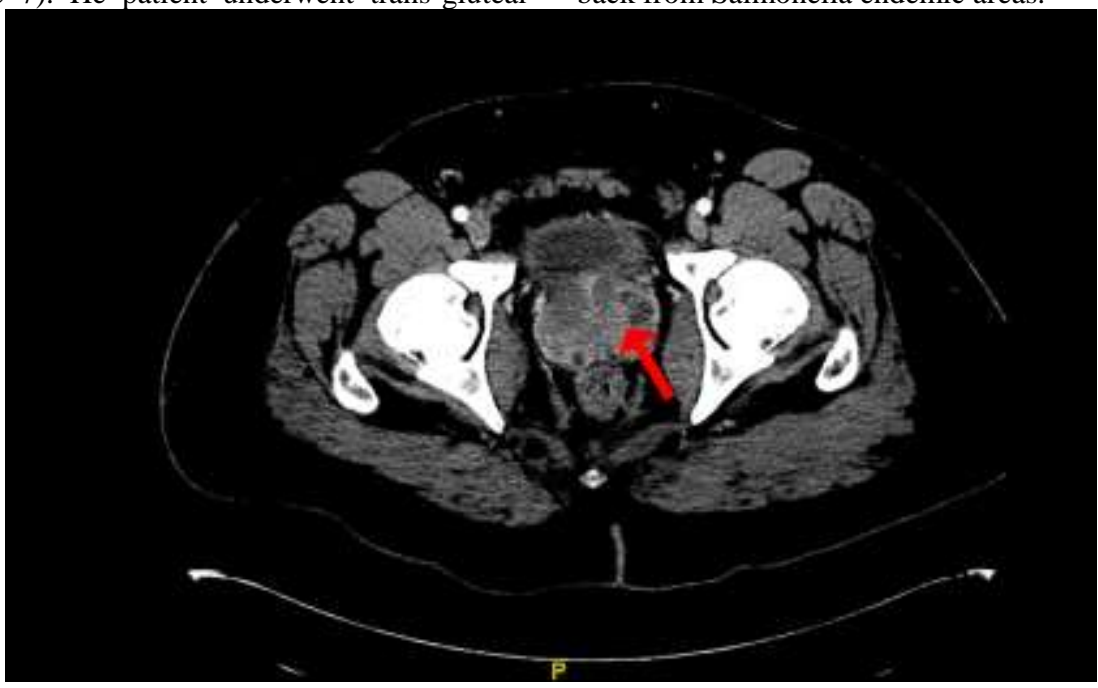

Figure 7 CT of the abdomen/pelvis with contrast demonstrating a moderately enlarged, heterogeneous appearing prostate gland with multiple hypoattenuating foci within it and surrounding inflammatory change concerning for multifocal prostatic abscesses.

Reproduced from: [25] Lin C, Patel S. Salmonella Prostatic Abscess in an Immunocompetent Patient. Cureus. 2020 July 10; $12(7)$ : e9122. doi:10.7759/cureus.9122 https://www.cureus.com/articles/34896-salmonella-prostatic-abscess-in-an-immunocompetent-patient under Creative Commons Attribution License which permits reproduction of contents and figures from the Cureus articles provided the original source is cited and credited.

Aust et al. [70] reported a case of tubercular prostatic abscess in a patient who had undergone intravesical bacillus Calmette-Guérin immunotherapy for bladder carcinoma in situ. His abscess did require surgical drainage and anti-tuberculous treatment.

Bour et al. [71] reported the diffusion and contrast-enhanced MRI appearance of five cases of granulomatous prostatitis (GP), non-specific (two cases) and infectious post-Bacillus Calmette-Guerin (BCG) therapy (three cases, with a tubercular abscess in two of them). All of the patients did have raising PSA levels and abnormal digital rectal examination (DRE) findings. Bour et al. [71] reported that a history of BCG treatment or acute prostatitis was present in four of the patients. Multiparametric MRI (T2W-MRI, DW-MRI and DCE-MRI) was undertaken before the biopsies were taken. The diagnosis of granulomatous prostatitis and tuberculous prostate abscess was confirmed by TRUS-guided biopsies in four cases and by transurethral resection in one case. MRI scan demonstrated a tumour-like appearance in three cases, an abscess-like appearance in one case and a combined tumour/abscess-like appearance in one case. Extra-prostatic fat was found to be infiltrated in three patients that simulated T3a disease. Histopathology examination of the specimens showed caseous necrosis when the MRI scan showed abcedation. Bour et al. [71] stated that the demonstration of occult tubercular abscesses in post-BCG GP could have therapeutic implications and MRI is useful prior to surgical or interventional drainage of large caseous abscesses.

Wang et al. [72] made the ensuing summating iterations about prostate abscess:

- $\quad$ Prostate abscess is an uncommon urological disease.
- Prostate abscess tends not to be easily diagnosed based upon the taking of history, clinical examination, laboratory test data, and radiology imaging studies.

- Patients who have prostate abscess and those patients who have prostate cancer could have simulating manifesting clinical signs and symptoms, such as lymph node enlargement and abnormal serum prostate-specific antigen values.

Wang et al. [72] reported a 67-year-old man who did not have any underlying diseases, who manifested with acute prostatitis. He underwent trans-rectal ultrasound (TRUS) scan 14 days after commencement of his antibiotic treatment which demonstrated a fluid-containing irregular mass. Doppler ultrasound scan demonstrated high vascularity encompassing the margin of the mass. Advanced prostate cancer with necrosis was found within tissue that was obtained from trans-urethral resection of the prostate (TURP). His symptoms did improve considerably pursuant to his undergoing TURP and he received androgen deprivation treatment in the outpatient clinic. Wang et al. [72] stated that even though ultrasound-guided needle aspiration of prostate abscess does remain the mainstream treatment for prostate abscess, TURP should be considered with regard to patients who have complicated prostate abscess or suspected carcinoma of the prostate gland.

\section{Conclusions and Recommendations}

- Prostate abscess is an uncommon complication that emanates from the acute and chronic infectious process of the prostate gland called (prostatitis) which is typified by a focal accumulation of 
purulent material within the prostate gland that affects the central and peripheral zone of the prostate gland.

- Prostate abscess tends to occur more commonly within the developing countries in comparison with developed countries.

- Prostate abscess tends to be more commonly associated with individuals who have diabetes mellitus, chronic liver disease including cirrhosis of the liver, chronic kidney diseases and urinary tract diseases as could be found in individuals who have staghorn calculus of the kidney, immunosuppression including HIV infection and transplant recipients and Salmonella has also been reported.

- Prostate abscess could be caused by bacteria or fungi and some of the organisms that have tended to be reported include: Escherichia coli, Klebsiella, Proteus, Pseudomonas, Enterobacteria, Salmonella, Brucella, Mycobacterium Tuberculosis, Nocardia.

- Development of tuberculous prostate abscess has been associated with intravesical instillation of bacillus Calmette Guerin as well as overt tuberculosis and hence in view of the non-specific nature of the symptoms a high index of suspicion is required.

- Development of salmonella prostate abscess has been associated with foreign travel to and back from Salmonella endemic area of the world by an individual dwelling in a non-Salmonella endemic area.

- $\quad$ Brucellosis prostate abscess can be acquired through drinking of fresh milk in a Brucellosis endemic area or being in contact with animals in a Brucellosis endemic area.

- Sexually transmitted prostate abscess can also occur.

- $\quad$ Prostate abscess does manifest with non-specific symptoms and hence a high index of suspicion is required to establish early diagnosis.

- Diagnosis of prostate abscess relies upon good clinical history taking, good and careful clinical examination and digital examination of bogginess within the prostate gland that is tender and fluctuant, laboratory test results with raised white blood cell count, and raised CRP, as well as lymphocytosis in cases of tuberculous prostate abscess. Radiology imaging including transrectal ultrasound scan of the prostate gland (the most commonly used option), computed tomography (CT) scan, and magnetic resonance imaging (MRI) scan, tend to illustrate the features and location of the abscess and radiology image-guided aspiration / drainage of the abscess tend to be undertaken as treatment and to obtain specimen for microscopy, culture and antimicrobial sensitivity to confirm the cause of the abscess and the antimicrobial agent to treat the patient with as well as radiology imaging is generally undertaken as part of careful regular followup assessment of individuals to confirm resolution of the abscess or recurrence / persistent abscess in order to provide further treatment.

- Treatment of prostate abscess depending upon the size of the abscess has varied depending upon the size of the abscess, the comorbidities of the patient, availability of an interventional radiologist, as well as availability of an interventional radiology department, the experience of the urologist/surgeon/clinician managing the patient, and the choice of the patient pursuant to discussion of the options of the treatment and the risks associated with the treatment.
- There is no global consensus opinion on the choice of treatment but some of the treatment options that have been used include: (a) antimicrobial treatment alone for small abscesses and careful assessment to ensure resolution and if there is persistence or recurrence of the abscess an alternative treatment of aspiration / drainage would be undertaken, (b) anti-microbial medicament plus aspiration of the abscess under radiology imaging by means of ultrasound scan most often or CT scan-guidance, or MRI scan guidance with utilization of appropriate antimicrobial medicament based upon the antibiotic sensitivity pattern of the cultured organism, (c) anti-microbial medicament plus insertion of drainage tube into the abscess cavity under radiology imaging by means of ultrasound scan most often or CT scan-guidance, or MRI scan guidance with utilization of appropriate antimicrobial medicament based upon the antibiotic sensitivity pattern of the cultured organism, (d) anti-microbial medicament plus trans urethral drainage and resection of the prostate abscess / deroofing of the abscess / modified resection of the prostate gland to deroof and drain the abscess with utilization of appropriate antimicrobial medicament based upon the antibiotic sensitivity pattern of the cultured organism, (e) on rare occasions utilization of open drainage of the abscess plus appropriate antimicrobial antimicrobial medicament based upon the anti-microbial sensitivity pattern of the cultured organism.

- It would be envisaged that there could be a scenario of nonavailability of radiology imaging facilities in some remote hospitals in some developing countries and in such places too apart from availability of facilities for routine haematology and biochemistry tests, facilities may not be available locally for culture of aspirates of the pus and the aspirate may need to be sent to the regional hospital or a national hospital that is far away and in such uncommon situations, the clinician may have to aspirate the abscess based upon the clinical examination finding of a tender soft fluctuant area within the prostate / bogginess under finger digital examination guidance and send the pus to a different hospital for culture and sensitivity and in such situations an empirical broad spectrum antibiotics need to be given and if the hospital is situated in a salmonella endemic area if the patient is not responding quickly to treatment then the clinician may need to have a multi-disciplinary team discussion to reflect upon treatment of salmonella infection empirically as well as chase the results of the pus culture from the regional / national hospital to ascertain the culture results including if the organism is a fugus. It may also be necessary for the patient to be sent to the nearest hospital where ultrasound scan facilities are available to undertake ultrasound scan of the prostate to assess for residual abscess / recurrent abscess pursuant to the emergency drainage of the prostate abscess.

- $\quad$ Recurrences of prostate abscess / persistence of prostate abscesses could occur following the different treatment options and the treatment of these would tend to depend upon the size and as well as number of recurrences or persistent lesions which can be treated based upon multi-disciplinary team discussion outcome and some of these could include: (a) recurrence / persistence after antibiotic treatment only may require either (i) aspiration of the abscess or (ii) insertion of a drain, or (iii) trans-urethral resection of the prostate / deroofing of the prostate abscess; (b) recurrence or persistence of the prostate abscess following aspiration of the abscess initially may require either (i) insertion of a drain under radiology imaging guidance or (ii) trans-urethral resection of prostate and deroofing of the abscess; (c) recurrence / persistence of the prostate abscess following trans-urethral resection / deroofing of the abscess especially a multi-septated-multi- 
compartmental abscess may require aspiration of residual / recurrent abscess under radiology image-guidance / insertion of a drain into the abscess under radiology imaging guidance.

- In cases of sepsis and septic shock, all hospitals should have intensive care / high dependency unit facilities to provide support for all patients and this should be associated with full discussion and involvement of the anaesthetists and the intensive care unit / high dependency unit team.

- If there are still hospitals globally that do not have facilities for radiology imaging including facilities for ultrasound scan machines, computed tomography scan machines, and magnetic resonance imaging machines then it would be appropriate for the urology department to liaise with other departments and the management team of the hospital to make a business case to provide radiology imaging facilities for the hospital as well as train staff to use the radiology imaging facilities.

- If there are no facilities for blood culture as well as urine culture in some hospitals in developing countries then concerted efforts should be made to provide such services and train staff in order to help facilitate good and excellent patient care.

\section{CONFLICT OF INTEREST - None}

\section{ACKNOWLEDGEMENTS}

\section{Acknowledgements to:}

- Journal of Investigative Medicine High Impact Case Reports. For granting permission for reproduction of figures and contents of their journal article under copyright: This article is distributed under the terms of the Creative Commons Attribution $4.0 \quad$ License (http://www.creativecommons.org/licenses/by/4.0/) which permits any use, reproduction and distribution of the work without further permission provided the original work is attributed as specified on the SAGE and Open Access pages (https://us.sagepub.com/en-us/nam/open-access-at-sage).

- I D Cases. For granting permission for reproduction of figures and contents of their journal article under copyright: Copyright (C) 2016 The Author(s) This is an open access article under the CC BY-NC-ND license (http://creativecommons.org/licenses/by-nc-nd/4.0/).

- West J Emerg Med. For granting permission for reproduction of figures and contents of their journal article under copyright this is an open access article distributed in accordance with the terms of the Creative Commons Attribution (CC BY 4.0) License. See: http://creativecommons.org/licenses/by-nc/4.0/.

- Cureus. For granting permission for reproduction of figures and contents of their journal articles under The Creative Commons Attribution License which permits reproduction of contents and figures from the Cureus articles provided the original source is cited and credited.

\section{References}

1. Reddivari AKR, Mehta P. Prostate Abscess. 2021 Jun 21. In: StatPearls [Internet]. Treasure Island (FL): StatPearls Publishing; 2021 Jan-. PMID: 31869127.

2. Faris G, Metz Y, Friedman B, Appel B, Stein A. [Prostatic abscess--diagnosis and treatment]. Harefuah. 2008 Jul;147(7):594-596, 663. Hebrew. PMID: 18814516.
3. Lee DS, Choe HS, Kim HY, Kim SW, Bae SR, Yoon BI, Lee SJ. Acute bacterial prostatitis and abscess formation. BMC Urol. 2016 Jul 7;16(1):38. doi: 10.1186/s12894-016-0153-7. PMID: 27388006; PMCID: PMC4936164.

4. Abdelmoteleb H, Rashed F, Hawary A. Management of prostate abscess in the absence of guidelines. Int Braz J Urol. 2017 SepOct;43(5):835-840. doi: 10.1590/S1677-5538.IBJU.2016.0472. PMID: 28379661; PMCID: PMC5678513.

5. Ahmed M. Elshal, Ahmed Abdelhalim, Tamer S. Barakat, Atallah A. Shaaban, Adel Nabeeh \& El-Housseiny Ibrahiem. Prostatic abscess: Objective assessment of the treatment approach in the absence of guidelines, Arab Journal of Urology. 2014;12:4, 262 268, DOI: 10.1016/j.aju.2014.09.002.

6. Granados EA, Caffaratti J, Farina L, Hocsman H. Prostatic abscess drainage: clinical-sonography correlation. Urol Int. 1992;48(3):358-361. PMID: 1589934.

7. Ludwig M, Schroeder-Printzen I, Schiefer HG, Weidner W. Diagnosis and therapeutic management of 18 patients with prostatic abscess. Urology. 1999 Feb;53(2):340-345. doi: 10.1016/s0090-4295(98)00503-2. PMID: 9933051.

8. Trauzzi SJ, Kay CJ, Kaufman DG, Lowe FC. Management of prostatic abscess in patients with human immunodeficiency syndrome. Urology. 1994 May;43(5):629-633. doi: 10.1016/0090-4295(94)90176-7. PMID: 8165764.

9. Meares EM Jr. Prostatic abscess. J Urol. 1986 Dec;136(6):12811282. doi: 10.1016/s0022-5347(17)45313-4. PMID: 3773105.

10. Oliveira P, Andrade JA, Porto HC, Filho JE, Vinhaes AF. Diagnosis and treatment of prostatic abscess. Int Braz J Urol. 2003 Jan-Feb;29(1):30-34. doi: 10.1590/s1677-55382003000100006. PMID: 15745464.

11. Barozzi L, Pavlica P, Menchi I, De Matteis M, Canepari M. Prostatic abscess: diagnosis and treatment. AJR Am J Roentgenol. 1998 Mar;170(3):753-757. doi: 10.2214/ajr.170.3.9490969. PMID: 9490969.

12. Galosi AB, Montironi R, Fabiani A, Lacetera V, Gallé G, Muzzonigro G. Cystic lesions of the prostate gland: an ultrasound classification with pathological correlation. J Urol. 2009 Feb;181(2):647-657. doi: 10.1016/j.juro.2008.10.006. Epub 2008 Dec 16. PMID: 19091354.

13. Aravantinos E, Kalogeras N, Zygoulakis N, Kakkas G, Anagnostou T, Melekos M. Ultrasound-guided transrectal placement of a drainage tube as therapeutic management of patients with prostatic abscess. J Endourol. 2008 Aug;22(8):17511754. doi: 10.1089/end.2008.0265. PMID: 18673079.

14. Jang K, Lee DH, Lee SH, Chung BH. Treatment of prostatic abscess: case collection and comparison of treatment methods. Korean J Urol. 2012 Dec;53(12):860-864. doi: 10.4111/kju.2012.53.12.860. Epub 2012 Dec 20. PMID: 23301131; PMCID: PMC3531640.

15. Basiri A, Javaherforooshzadeh A. Percutaneous drainage for treatment of prostate abscess. Urol. J. 2010 Fall;7(4):278-280. PMID: 21170860.

16. Shah H. V323Transurethral holmium laser deroofing of prostatic abscess: description of technique and early results. Abstract. J Urol. 2010;183(45):e128.

17. Puzyrenko A, Iczkowski KA. Abscess. PathologyOutlines.com w ebsite.. Accessed September 20th, 2021.

18. Johnson MG, Caplan-Shaw CE, McMacken M. Tuberculous prostate abscesses in an immunocompetent patient: A dramatic presentation of disseminated tuberculosis. Germs. 2014 Jun;4(2):41-45. DOI: 10.11599/germs.2014.1054. PMID: 24967218; PMCID: PMC4067608.

19. Lee $\mathrm{P}$, Ong T, Dayangku Norlida A. Tuberculous prostatic abscess in an immunocompromised patient. Malaysian Family Physician : 
the Official Journal of the Academy of Family Physicians of Malaysia. 2010 ;5(3):145-147. PMID: 25606207; PMCID: PMC4170414.

20. Duarte Ojeda JM, García Luzón A, Carrero VM, Vázquez S, Calahorra L, Passas J, Leiva O. Absceso prostático tuberculoso en paciente con SIDA [Tuberculous prostatic abscess in a patient with AIDS]. Actas Urol. Esp. 1995 Sep;19(8):655-661. Spanish. PMID: 8669335.

21. Doo SW, Kim JH, Yang WJ, et al. A case of tuberculous prostatitis with abscess. The World Journal of Men's Health. 2012 Aug;30(2):138-140. DOI: 10.5534/wjmh.2012.30.2.138. PMID: 23596601 ; PMCID: PMC3623522.

22. Baral S, Chhetri R K, Gyawali M, Thapa N, Mahato R, Sharma R, Dahal P. Prostate tuberculosis complicated by huge prostatic abscess: A rare case report from Nepal, International Journal of Surgery Case Reports. 2020; 77: 152 - 156. ISSN 2210-2612,

23. Alenazi A H, Alfhaidi F M, Aljumaah A A S, Alenzi M J, AlOtaibi M N. A Rare Case of Prostatic Abscess Caused by Brucella Organisms: First Report in Saudi Arabia. Urology Annals. 2018 Jan-Mar; 10(1): 106 - 107. DOI: 10.4103/UA.UA_67_17

24. Dakdouki GK, Al Awar GN. Brucella prostatic abscess: first case report. Scandinavian Journal of Infectious Diseases. 2005 ;37(9):692-694. DOI: 10.1080/00365540410021162. PMID: 16126573.

25. Lin C, Patel S. Salmonella Prostatic Abscess in an Immunocompetent Patient. Cureus. 2020 July 10; 12(7): e9122. doi:10.7759/cureus.9122

26. Learmonth DJ, Philp NH. Salmonella prostatic abscess. Br J Urol. 1988 Feb;61(2):163. doi: 10.1111/j.1464-410x.1988.tb05070.x. PMID: 3280082.

27. Giessing, Markus; Baumgart, Egbert; Lenk, Severin; Loening, Stefan A Salmonella typhimurium prostatic abscess: a rare cause of persisting dysuria in an AIDS patient, AIDS. 2003 Feb 14; 17( 3) 449-450.

28. Tiwari P, Pal DK, Tripathi A, Kumar S, Vijay M, Goel A, Sharma P, Dutta A, Kundu AK. Prostatic abscess: diagnosis and management in the modern antibiotic era. Saudi J Kidney Dis Transpl. 2011 Mar;22(2):298-301. PMID: 21422629.

29. Brede CM, Shoskes DA. The etiology and management of acute prostatitis. Nat Rev Urol. 2011 Apr;8(4):207-212. doi: 10.1038/nrurol.2011.22. Epub 2011 Mar 15. PMID: 21403661.

30. Susaníbar Napurí LF, Simón Rodríguez C, López Martín L, Monzó Gardinier J, Cabello Benavente R, González Enguita C. Prostatic abscess: diagnosis and treatment of an infrequent urological entity. Arch Esp Urol. 2011 Jan-Feb;64(1):62-66. English, Spanish. PMID: 21289388.

31. Schneider H, Ludwig M, Hossain HM, Diemer T, Weidner W. The 2001 Giessen Cohort Study on patients with prostatitis syndrome--an evaluation of inflammatory status and search for microorganisms 10 years after a first analysis. Andrologia. 2003 Oct;35(5):258-262. PMID: 14535851.

32. Yamamoto M, Hibi H, Miyake K. Prostate-specific antigen levels in acute and chronic bacterial prostatitis. Hinyokika Kiyo. 1993 May;39(5):445-449. PMID: 7686714.

33. Thornhill BA, Morehouse HT, Coleman P, Hoffman-Tretin JC. Prostatic abscess: CT and sonographic findings. AJR Am J Roentgenol. $1987 \quad$ May;148(5):899-900. doi: 10.2214/ajr.148.5.899. PMID: 3554922.

34. Singh P, Yadav MK, Singh SK, Lal A, Khandelwal N. Case series: Diffusion weighted MRI appearance in prostatic abscess. Indian J Radiol. Imaging. 2011 Jan;21(1):46-48. doi: 10.4103/09713026.76054. PMID: 21431033; PMCID: PMC3056370.

35. Jana T, Machicado JD, Davogustto GE, Pan JJ. MethicillinResistant Staphylococcus aureus Prostatic Abscess in a Liver
Transplant Recipient. Case Rep Transplant. 2014;2014:854824. doi: 10.1155/2014/854824. Epub 2014 Oct 20. PMID: 25389507; PMCID: PMC4217349.

36. Carroll DE, Marr I, Huang GKL, Holt DC, Tong SYC, Boutlis CS. Staphylococcus aureus Prostatic abscess: a clinical case report and a review of the literature. BMC Infect Dis. $2017 \mathrm{Jul}$ 21;17(1):509. doi: 10.1186/s12879-017-2605-4. PMID: 28732492; PMCID: PMC5521102.

37. Liao, CY., Yang, YS., Yeh, YC. et al. Invasive liver abscess syndrome predisposed by Klebsiella pneumoniae related prostate abscess in a nondiabetic patient: a case report. BMC Res Notes 9, 395 (2016).

38. Bastide C, Carcenac A, Arroua F, Rossi D. Prostatic abscess due to Candida tropicalis. Prostate Cancer Prostatic Dis. 2005;8(3):296-297. doi: 10.1038/sj.pcan.4500809. Erratum in: Prostate Cancer Prostatic Dis. 2009 Dec;12(4):382. Arrova, F [corrected to Arroua, F]. PMID: 15953933.

39. Somuncu I, Sağlam M, Yağci S, Tahmaz L, Taşar M, Ors F. Multiloculated prostate abscess: treatment with transrectal ultrasound guided transrectal needle aspiration and lavage with the saline and antibiotic. Clin Imaging. 2003 Jul-Aug;27(4):251255. doi: 10.1016/s0899-7071(02)00546-6. PMID: 12823920.

40. Chou YH, Tiu CM, Liu JY, Chen JD, Chiou HJ, Chiou SY, Wang JH, Yu C. Prostatic abscess: transrectal color Doppler ultrasonic diagnosis and minimally invasive therapeutic management. Ultrasound Med Biol. 2004 Jun;30(6):719-724.

41. Varkarakis J, Sebe P, Pinggera GM, Bartsch G, Strasser H. Threedimensional ultrasound guidance for percutaneous drainage of prostatic abscesses. Urology. 2004 Jun;63(6):1017-1020; discussion 1020. doi: 10.1016/j.urology.2003.12.040. PMID: 15183937.

42. Basiri A, Javaherforooshzadeh A. Percutaneous drainage for treatment of prostate abscess. Urol J. 2010 Fall;7(4):278-280. PMID: 21170860

43. Percutaneous Drainage for Treatment of Prostate Abscess Abbas Basiri, Ahmad Javaherforooshzadeh Urol J. 2010;7:278-280.

44. Savarirayan S, Shenykin Y, Gerard P, Wise GJ. Staphylococcus periprostatic abscess: an unusual cause of acute urinary retention. Urology. 1995;46:573-574.

45. Bachor R, Gottfried HW, Hautmann R. Minimal invasive therapy of prostatic abscess by transrectal ultrasound-guided perineal drainage. Eur Urol. 1995;28:320-324.

46. Ludwig M, Schroeder-Printzen I, Schiefer HG, Weidner W. Diagnosis and therapeutic management of 18 patients with prostatic abscess. Urology. 1999;53: 340-345

47. Meares EM, Jr. Prostatic abscess. J Urol. 1986;136:1281-1282.

48. Collado A, Palou J, Garcia-Penit J, Salvador J, de la Torre P, Vicente J. Ultrasound-guided needle aspiration in prostatic abscess. Urology. 1999;53: 548-552.

49. [Lim JW, Ko YT, Lee DH, et al. Treatment of prostatic abscess: value of transrectal ultrasonographically guided needle aspiration. J Ultrasound Med. 2000;19:609-617.

50. El-Shazly M, El-Enzy N, El-Enzy K, Yordanov E, Hathout B, Allam A. Transurethral drainage of prostatic abscess: points of technique. Nephrourol Mon. 2012 Spring;4(2):458-461.

51. Suman Baral, Raj Kumar Chhetri, Milan Gyawali, Neeraj Thapa, Ranjit Mahato, Rupesh Sharma, Prahar Dahal, Prostate tuberculosis complicated by huge prostatic abscess: A rare case report from Nepal, International Journal of Surgery Case Reports. 2020; 77: 152 - 156. ISSN 2210-2612,

52. Kim JH, Yang WJ, Kim TH. Klebsiella pneumonia-induced prostate abscess: How to work it up? Can Urol Assoc J. 2014 Nov;8(11-12):E841-844. doi: 10.5489/cuaj.2155. Erratum in: Can 
Urol Assoc J. 2015 Jan-Feb;9(1-2):E55. PMID: 25485013; PMCID: PMC4250250.

53. Ullah A, Khakwani Z, Mehmood H. Prostate Abscess Caused by Community-Acquired Methicillin-Resistant Staphylococcus aureus. J Investig Med High Impact Case Rep. 2018 Jul 18;6:2324709618788899. doi: 10.1177/2324709618788899. PMID: 30038913; PMCID: PMC6052489.

54. Deshpande A, Haleblian G, Rapose A. Prostate abscess: MRSA spreading its influence into Gram-negative territory: case report and literature review. BMJ Case Rep. 2013 Mar 25;2013:bcr2013009057. doi: 10.1136/bcr-2013-009057. PMID: 23531939; PMCID: PMC3618833

55. Sakamaki I, Ueno A, Kawasuji H, Miyajima Y, Kawago K, Hishikawa Y, Ikehata Y, Fujiuchi Y, Kitamura H, Yamamoto Y. Prostate abscess caused by Nocardia farcina. IDCases. 2019 Sep 9;18:e0640. doi: 10.1016/j.idcr.2019.e00640. PMID: 31692509; PMCID: PMC6804916.

56. Scorey H, Daniel S. Nocardia farcinica bacteraemia presenting as a prostate abscess. I D Cases. 2016. 5: 24-26. ISSN 2214-2509,

57. Goyal NK, Goel A, Sankhwar S, Dalela D. Transurethral resection of prostate abscess: is it different from conventional transurethral resection for benign prostatic hyperplasia? ISRN Urol. 2013 Jun 11;2013:109505. doi: 10.1155/2013/109505. PMID: 23840969; PMCID: PMC3693178.

58. Gilmore P E, Baird A D, Javle P M. Prostate Abscess: A Rare Complication of Brachytherapy for Prostate Cancer. Ann R Coll Engl. 2009 May; 91(4): W12-W14.

59. Bae GB, Kim SW, Shin BC, Oh JT, Do BH, Park JH, Lee JM, Kim NS. Emphysematous prostatic abscess due to Klebsiella pneumoniae: report of a case and review of the literature. J Korean Med Sci. 2003 Oct;18(5):758-60. doi: 10.3346/jkms.2003.18.5.758. PMID: 14555835; PMCID: PMC3055114.

60. Chuang L H, Song H S, Lee S C, Lai C C, Ku W C. Endogenous Klebsiella pneumoniae endophthalmitis associated with prostate abscess: case report. Chang Gung Medical Journal, 01 Apr 2000, 23(4):240-245. PMID: 10902231

61. Stafford P, Prybys KM. Pyocystis and prostate abscess in a hemodialysis patient in the emergency department. West J Emerg Med. 2014 Sep;15(6):655-8. doi: 10.5811/westjem.2014.5.22317. PMID: 25247036; PMCID: PMC4162722.

62. Walker B, Heidel E, Shorman M. Clinical Characteristics and Outcome of Staphylococcus aureus Prostate Abscess From Ten Years of Experience at a Tertiary Care Center, Open Forum Infectious Diseases. 2019 Oct; 6(10): ofz372,
63. Arrabal-Polo MA, Jimenez-Pacheco A, Arrabal-Martin M. Percutaneous drainage of prostatic abscess: case report and literature review. Urol Int. 2012;88(1):118-20. doi: 10.1159/000330934. Epub 2011 Sep 21. PMID: 21934282.

64. Quraishi MK, Phan YC, Asaad W, Lynn N. Prostatic abscess: a rare complication of staghorn calculi. BMJ Case Rep. 2018 Feb 1;2018:bcr2017222917. doi: 10.1136/bcr-2017-222917. PMID: 29391357; PMCID: PMC5812406

65. Dakdouki GK, Al Awar GN. Brucella prostatic abscess: first case report. Scand J Infect Dis. 2005;37(9):692-694. doi: 10.1080/00365540410021162. PMID: 16126573.

66. Alenazi AH, Alfhaidi FM, Aljumaah AAS, Alenzi MJ, AlOtaibi MM. A rare case of prostatic abscess caused by Brucella organisms: First report in Saudi Arabia. Urol Ann. 2018 JanMar;10(1):106-107. doi: 10.4103/UA.UA_67_17. PMID: 29416286; PMCID: PMC5791446

67. S Dalal, R Sahu, Nityasha, R Dahiya. Prostatic Abscess: Easy to diagnose, difficult to treat. The Internet Journal of Surgery. 2009. 23(2):

68. Oliveira P, Andrade JA, Porto HC, Filho JE, Vinhaes AF. Diagnosis and treatment of prostatic abscess. Int Braz J Urol. 2003 Jan-Feb;29(1):30-4. doi: 10.1590/s1677-55382003000100006. PMID: 15745464.

69. Flannery MT, Humphrey D. Case report of a prostatic abscess with a review of the literature. Case Rep Med. 2012;2012:430657. doi: 10.1155/2012/430657. Epub 2012 Aug 30. PMID: 22969809; PMCID: PMC3437292.

70. Gupta S, Liu-Young G, Mahnensmith Rex, Topal, Jeffrey E. Prostatic Abscess With Candida glabrata Treated With Micafungin. Infectious Diseases in Clinical Practice. 2008 Nov; 16(6): 387 - 389. doi: 10.1097/IPC.0b013e31816613f1.

71. Aust TR, Massey JA. Tubercular prostatic abscess as a complication of intravesical bacillus Calmette-Guérin immunotherapy. Int J Urol. 2005 Oct;12(10):920-1. doi: 10.1111/j.1442-2042.2005.01183.x. PMID: 16323989.

72. Bour L, Schull A, Delongchamps N B, Beuvon F, Muradyan N, Legmann P, Cornud F. Multiparametric MRI features of granulomatous prostatitis and tubercular prostate abscess. Diagnostic and Interventional Imaging. 2013; 94(1): 84-90. ISSN 2211-5684,

73. Wang S-F, Hsieh T-S. Advanced prostate cancer presenting as a huge abscess, Urological Science. 2014; 25(4): 126-128. ISSN 1879-5226.
This work is licensed under Creative Commons Attribution 4.0 License
Ready to submit your research? Choose Auctores and benefit from:

$>$ fast, convenient online submission

$>$ rigorous peer review by experienced research in your field

$>$ rapid publication on acceptance

$>$ authors retain copyrights

$>$ unique DOI for all articles

$>$ immediate, unrestricted online access

At Auctores, research is always in progress.

Learn more https://www.auctoresonline.org/journals/archives-of-medical-casereports-and-case-study 